\title{
Hybrid Access Femtocells in Overlaid MIMO Cellular Networks with Transmit Selection under Poisson Field Interference
}

\author{
Amr A AbdelNabi, Fawaz S. Al-Qahtani, Redha M. Radaydeh, and Mohammed Shaqfeh
}

\begin{abstract}
This paper analyzes the performance of hybrid control-access schemes for small cells (such as femtocells) in the context of two-tier overlaid cellular networks. The proposed hybrid access schemes allow for sharing the same downlink resources between the small-cell network and the original macrocell network, and their mode of operations are characterized considering post-processed signalto-interference-plus-noise ratios (SINRs) or pre-processed interference-aware operation. The work presents a detailed treatment of achieved performance of a desired user that benefits from MIMO arrays configuration through the use of transmit antenna selection (TAS) and maximal ratio combining (MRC) in the presence of Poisson field interference processes on spatial links. Furthermore, based on the interference awareness at the desired user, two TAS approaches are treated, which are the signal-tonoise (SNR)-based selection and SINR-based selection. The analysis is generalized to address the cases of highlycorrelated and un-correlated aggregated interference on different transmit channels. In addition, the effect of delayed TAS due to imperfect feedback and the impact of arbitrary TAS processing are investigated. The analytical results are validated by simulations, to clarify some of the main outcomes herein.
\end{abstract}

Index Terms-Hybrid access schemes, femtocells, twotier cells, MIMO, transmit selection, Poisson Interference, MRC, imperfect feedback, TAS/MRC, stochastic geometry, outage probability, average error rate, asymptotic performance.

\section{INTRODUCTION}

The rapidly growing demands for high data rate services in dense networks, which exceeds the current capacity of cellular systems, necessitates new technologies that fulfill the growth on network capacity and coverage. In

Amr A AbdelNabi is with IMDEA networks, 28918, Spain, Email: amr.abdelnabi@imdea.org.

F. Al-Qahtani, and Mohammed Shaqfeh are with Electrical \& Computer Engineering Program, Texas A\&M University at Qatar, Doha, Qatar, E-mail: \{fawaz.al-qahtani, mohammed.shaqfeh\}@qatar.tamu.edu.

R. M. Radaydeh is with Computer, Electrical, and Mathematical Sciences and Engineering Division at King Abdullah University of Science and Technology (KAUST), Thuwal, 23955-6900, KSA, Email: redha.radaydeh@gmail.com.

This work is supported by Qatar National Research Fund (QNRF) grant through National Priority Research Program (NPRP) No. 81545-2-657. QNRF is an initiative of Qatar Foundation. particular, there has been a great interest to enhancing the capacity of the current cellular standards, such as the 3GPP Long-Term Evolution (LTE) standard, in indoor environments. However, providing high data rate coverage indoors cannot be achieved with the current macrocell network architecture due to high penetration losses. Among the techniques that were proposed to solve this particular problem are distributed antennas [1] and microcell/macrocell architecture [2]. However, such technologies require a relatively high operation cost.

On the other hand, the use of small, low power and low cost access points (known also as femtocells) that are deployed by either users or operators to enhance capacity and coverage has been of interest recently. The co-existence of femtocells and original macrocells, which create a two-tier cellular network, result in many technical challenges, such as the increase in cross-tier interference and co-tier interference [3]. Cross-tier interference is observed between different tiers while co-tier interference exists in the same tier.

There can be many implementation approaches of femtocells within the cellular network. For instance, dedicated spectrum bands (e.g., subcarriers of the orthogonal frequency division multiplexing (ODFM)) may be used for femtocells. Consequently, cross-tier interference can be eliminated on the expense of wasting spectrum resources. Alternatively, available spectrum bands can be jointly utilized and shared by the macrocells and femtocells, which requires power control for pilot and data signals to mange interference [4]. The work in [5] proposed a different model based on cognitive operation wherein a femtocell senses the medium and changes its subchannels accordingly. Moreover, the access control scheme used at femtocells can significantly affect the level of interference. Among these access protocols, closed access, open access, and hybrid access has been adopted [6]. The closed access is known as closed subscriber group (CSG), where only authorized femtocell user can access the femtocell resources. On the other hand, the open access, which is known as open subscriber group (OSG), permits cellular users to use resources of a nearby femtocell as long as they are within the femtocell coverage. Finally, the hybrid access can be designed to allow for a planned and limited sharing of femtocell 
resources by unauthorized cellular users.

The use of multiple input multiple output (MIMO) systems has been shown to increase the channel diversity and reduce the effect of fading [7,8]. They can be also used to support higher data rates through spatial multiplexing [8]. However, one of the difficulties of MIMO systems is the need of multiple radio frequency (RF) chains, which increases the implementation cost significantly. To overcome this, transmit antenna selection (TAS) has been proposed to reduce the number of RF chains without losing the full diversity of MIMO system [9]. The use of TAS is usually accompanied by maximal ratio combining (MRC) at the receiver, and in point-to-point MIMO, TAS/MRC has been shown to achieve full transmit/receive diversity [10]-[12]. In this case, a transmit antenna that maximizes the MRC postprocessed signal-to-noise ratio (SNR) at the receiver is selected for the scenario of SNR-based selection [13], or that maximizes the post-processed signal-to-interferencenoise ratio (SINR) is selected for the scenario of SINRbased selection [14].

One important aspect that should be taken into consideration in the analysis and design of femtocells that reuse the same resources of the macrocell is the model of interference. In this context, having fixed number of interferers at fixed and known locations would be a hard assumption in practice. Alternatively, in order to cope with the irregular access points locations in heterogeneous networks, stochastic geometry can be a promising approach to characterize interference. In this model, which includes $\alpha$-Stable probability distribution [15], interferers are assumed to be distributed randomly according to a Poisson Point Process (PPP). In [16], PPP was used to model the interference from macrocell base stations, while in [17], it modelled cross-tier interference from femtocells. In [18], it was used to model cochannel interference in ad-hoc networks. In this paper, we adopt the PPP field interference to model interference processes on spatial MIMO links.

This paper investigates a newly formulated problem that integrates different processing designs, operation and network model conditions in the context of twotier cellular networks. Our objective is to analyze the downlink performance of hybrid control-access scheme for spectrum-shared two-tier cellular MIMO networks with the use of TAS/MRC in the presence of random interference sources that follow PPP on spatial links. The contributions of this paper are summarized as follows:

- The mode of operation of proposed SINR-based Post-TAS hybrid access schemes and Pre-TAS interference-aware hybrid access schemes are described and formulated.
- New analytical results for the outage probability and average error rate of a desired user are developed with the use of the proposed hybrid access scheme and PPP interference field on spatial links. These results consider different interference conditions at the serving access point and different transmit antenna selection algorithms. Specifically, two TAS approaches are treated, which are the signal-to-noise (SNR)-based selection and signal-to-interferenceplus-noise ratio (SINR)-based selection.

- The analysis covers two possible scenarios in which the observed interference sources at the desired user station are either highly-correlated or un-correlated as seen on transmit channels of the serving station.

- The developed results demonstrate the performance enhancement of SINR-based selection with respect to the SNR-based selection. Moreover, asymptotic approximations for the considered performance metrics are presented, which enable the characterization of the key performance indicators, such as the diversity gain and coding gain, sizes of transmit and receive array, and antenna selection algorithm, on the achieved downlink performance at a desired user.

- The effect of imperfect TAS due to imperfect feedback channel and the impact of arbitrary TAS processing on the aforementioned performance measures are investigated and compared with the idealized operation scenario.

The rest of the paper is organized as follows. Section II introduces the system model, Pre-TAS SINR formulations and interference models. The proposed hybrid control-access scheme is discussed in Section III. The performance analysis of the adopted system and interference models with the hybrid access scheme using SNRbased TAS is presented in Section IV, which is followed by asymptotic performance results in Section V, and then the investigation of the effect of feedback delay and arbitrary TAS processing in Section VI. The analysis of performance measures with the use of SINR-based TAS is then treated in Section VII, which will be followed by selected numerical and simulation results in Section VIII. Finally, concluding remarks are provided in Section IX.

Mathematical Notations: The notations used herein are as follows. Bold lower/upper case symbols denote vectors/matrices, respectively. Use of $(.)^{T}$ for transpose, $(.)^{H}$ conjugate transpose, $\|$.$\| for norm, and (\cdot)$ for binomial coefficient. Use of $\mathbb{E}\{$.$\} to denote the expectation$ operator. The term $f_{x}($.$) denotes the Probability density$ function (PDF) of the random variable $x . \mathcal{F}_{x}(\gamma)=$ 
$\operatorname{Pr}(x<\gamma)$ is the Cumulative Distribution Function (CDF) of random variable $x . \mathcal{M}_{x}(s)=\mathbb{E}\{-s x\}$ is the moment generating function for the random variable $x$. $\mathcal{L}^{-1}\{\}$ denotes the inverse Laplace transform. $\Gamma($.$) is the$ complete gamma function. $\gamma($.$) is the incomplete gamma$ function.

The Fox H-function properties used throughout the paper are summarized as:

Definition 1: The identity property of Fox H-Function is expressed as [19, Eq 1.125]

$$
H_{0,1}^{1,0}\left[\left.Z\right|_{(b, B)}\right]=B^{-1} Z^{\frac{b}{B}} \exp \left(-Z^{\frac{-1}{B}}\right)
$$

Definition 2: The reciprocal property of Fox H-Function is given as [19, $\mathrm{Eq} .1 .58$ ]

$$
H_{p, q}^{m, n}\left[\left.Z\right|_{\left(b_{q}, B_{q}\right)} ^{\left(a_{p}, A_{p}\right)}\right]=H_{q, p}^{n, m}\left[\left.\frac{1}{Z}\right|_{\left(1-a_{p}, A_{p}\right)} ^{\left(1-b_{q}, B_{q}\right)}\right]
$$

Definition 3: The integral identity of Fox $\mathrm{H}$-function is defined as $[19$, P. 60]

$$
\begin{aligned}
& \int_{0}^{\infty} x^{\alpha-1} H_{u, v}^{s, t}\left[\left.\sigma x\right|_{\left(d_{v}, D_{v}\right)} ^{\left(c_{u}, C_{u}\right)}\right] H_{p, q}^{k, n}\left[\left.w x^{r}\right|_{\left(b_{q}, B_{q}\right)} ^{\left(a_{p}, A_{p}\right)}\right] d x \\
& =\sigma^{-\alpha} H_{p+v, q+u}^{k+t, n+s}\left[\frac{w}{\sigma^{r}} \mid \begin{array}{l}
\left(\bar{a}_{p+v}, \bar{A}_{p+v}\right) \\
\left(\bar{b}_{q+u}, \bar{B}_{q+u}\right)
\end{array}\right],
\end{aligned}
$$

where $r>0,\left(\bar{a}_{p+v}, \bar{A}_{p+v}\right)=\left\{\left(a_{n}, A_{n}\right),(1-\right.$ $\left.\left.d_{v} \quad-\quad \alpha D_{v}, r D_{v}\right),\left(a_{n+1}, A_{n+1}\right) \ldots\left(a_{p}, A_{p}\right)\right\}$, and $\left(\bar{b}_{q+u}, \bar{B}_{q+u}\right)=\left\{\left(b_{k}, B_{k}\right),\left(1-c_{u}-\right.\right.$ $\left.\left.\alpha C_{u}, r C_{u}\right),\left(b_{k+1}, B_{k+1}\right) \ldots\left(b_{q}, B_{q}\right)\right\}$.

Definition 4: The inverse Laplace property of Fox Hfunction is defined as [19, P.51]

$$
\begin{aligned}
& \mathcal{L}^{-1}\left\{t^{-\rho} H_{p, q}^{k, n}\left[\left.a t^{\sigma}\right|_{\left(b_{q}, B_{q}\right)} ^{\left(a_{p}, A_{p}\right)}\right] ; t ; x\right\} \\
& =x^{\rho-1} H_{p+1, q}^{k, n}\left[\left.a x^{-\sigma}\right|_{\left(b_{q}, B_{q}\right)} ^{\left(a_{p}, A_{p}\right),(\rho, \sigma)}\right] .
\end{aligned}
$$

\section{System AND InTERfERENCE Models}

This section contains three main parts. The first part discusses the adopted system model, and the second part presents the network interference models and the corresponding Pre-TAS SINR formulations. The third part describes the resulting SINR at the desired user station following TAS operation based on different criteria.

\section{A. System Model}

An overlaid ${ }^{1}$ two-tier cellular network is considered, which contains two types of access points (or base stations) as shown in Fig. 1. Each tier models the access points of a particular class, such as those of macrocells and femtocells. The BSs across tiers may differ in terms of the transmit power, the supported data rate and their spatial density. Both tiers share the same band. Throughout the remaining discussions, the

\footnotetext{
${ }^{1}$ Here, the term "overlay" denotes the coexistence of femtocell within the marcocell coverage.
}

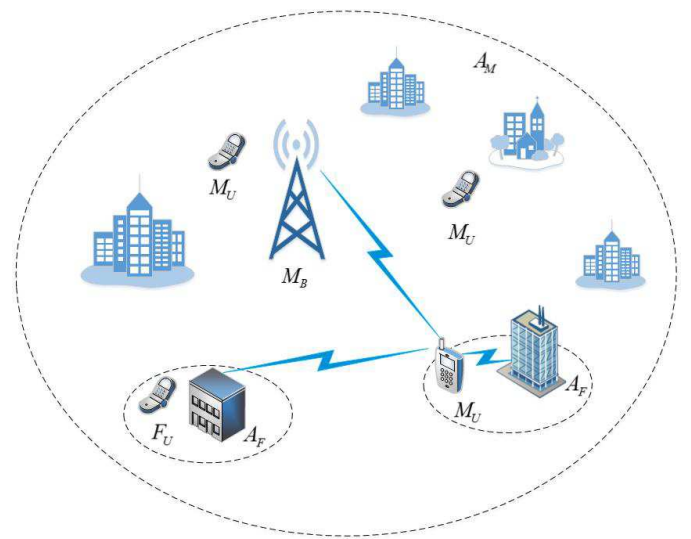

Fig. 1: Adopted hybrid access scheme.

notations $t=\left\{\mathrm{M}_{B}, \mathrm{~F}_{B}\right\}$ and $k=\left\{\mathrm{M}_{U}, \mathrm{~F}_{U}\right\}$ are used as defined in Table I.

The macrocell base station $\mathrm{M}_{B}$ can serve arbitrary number of macrocell users in its coverage area $A_{\mathrm{M}_{B}}$. The area $A_{\mathrm{M}_{B}}$ is overlaid by arbitrary number of femtocell access points, which can provide service to an arbitrary number of femtocell users as well as controlled number of macrocell users (according to the proposed hybrid access at each femtocell access point $\mathrm{F}_{B}$ ) in their coverage area $A_{\mathrm{F}_{B}}$.

Although the formulations presented below are directly applicable for any user identity, it is considered that a specific multi-antenna $\mathrm{M}_{U}$, which is authorized to receive downlink service from $\mathrm{M}_{B}$, enters the coverage area of an $\mathrm{F}_{B}$. Then, with the aim to extend the coverage while minimizing the interference experienced at $\mathrm{M}_{U}$, the hybrid access capability of $\mathrm{F}_{B}$ is exploited for an efficient link selection to serve $\mathrm{M}_{U}$. In this case, the resources available at $\mathrm{F}_{B}$ are distributed among authorized femtocell users and unauthorized $\mathrm{M}_{U}$ users, if applicable.

\section{B. Interference Models and Pre-TAS SINR Formulations}

It is assumed that $\mathrm{M}_{U}$ users, $\mathrm{F}_{U}$ users, and $\mathrm{F}_{B}$ access points, are assumed to follow stationary Poisson Point Processes (PPPs), where the PPP $\Phi_{\nu}$, with $\nu=\{t, k\}$, has density $\lambda_{\nu}$ with an average number of nodes $\lambda_{\nu} A_{\nu}$ in area $A_{\nu}$. An access point is assumed to have $N_{t}$ transmit antennas, whereas a user receive station is assumed to have $L_{k}$ receive antennas. The channel gain matrix $\mathbf{H}_{t, k}$ has $N_{t} \times L_{k}$ complex-valued channel gain entries with $h_{t, i, k, r}$, which can be expressed as $h_{t, i, k, r}=$ $\alpha_{t, i, k, r} e^{-j \phi_{t, i, k, r}}\left|d_{t, k}\right|^{-\frac{v}{2}}$. The small-scale fading channel envelopes on MIMO links are modelled independent and identical (i.i.d.) Rayleigh processes.

The composite received signal at user $k$ receive array that has a size $L_{k}$, when the $i$ th transmit antenna at access 


\begin{tabular}{|c|c|}
\hline Notation & Description \\
\hline$M_{B}, F_{B}, M_{U}, F_{U}$ & $\begin{array}{l}\text { Macrocell base station, femtocell base station, } \\
\text { macrocell user, and femtocell user, respectively. }\end{array}$ \\
\hline$t$ & $\begin{array}{l}\text { Macrocell base station and femtocell base } \\
\text { station, i.e. } t \in\left\{M_{B}, F_{B}\right\}\end{array}$ \\
\hline$k$ & $\begin{array}{l}\text { Macrocell user and femtocell user, i.e. } \\
\qquad k \in\left\{M_{U}, F_{U}\right\}\end{array}$ \\
\hline$A_{M_{B}}, A_{F_{B}}$ & $\begin{array}{l}\text { Coverage area of macrocell base station, and } \\
\text { femtocell base station, respectively. }\end{array}$ \\
\hline$\Phi_{\nu}$ & Poisson Point Process (PPPs) with $\nu=\{t, k\}$. \\
\hline$\lambda_{\nu}$ & Density of nodes. \\
\hline$N_{t}$ & Number of transmit antennas. \\
\hline$L_{k}$ & Number of receive antennas. \\
\hline $\mathbf{H}_{t, k}$ & $\begin{array}{c}\text { Channel gains matrix between access point } t \\
\text { and user } k \text {. }\end{array}$ \\
\hline$h_{t, i, k, r}$ & $\begin{array}{l}\text { Complex-valued channel gain observed on the } \\
t \rightarrow k \text { link between the } i \text { th transmit antenna } \\
\text { and the } r \text { th receive antenna with } \\
\mathbb{E}\left\{\alpha_{t}^{2}\right.\end{array}$ \\
\hline$\alpha_{t, i, k, r}$ & Small-scale fading channel envelope. \\
\hline$\phi_{t, i, k, r}$ & Small-scale fading channel phase. \\
\hline$v$ & Path loss exponent. \\
\hline$d_{t, k}$ & Separation distance of the $t \rightarrow k$ link. \\
\hline $\mathbf{z}_{t, i, k}$ & $\begin{array}{l}\text { Composite received signal at user } k \text { receive } \\
\text { array that has a size } L_{k} \text {, when the } i \text { th transmit } \\
\text { antenna at access point with } t \text { is used. }\end{array}$ \\
\hline $\mathbf{h}_{t, i, k}$ & $\begin{array}{l}\text { Column vector of i.i.d. channel gains } \\
\text { associated with desired data symbol. }\end{array}$ \\
\hline $\mathbf{g}_{t, i, k, m}$ & $\begin{array}{l}\text { Column vector of the } m \text { th source of } \\
\text { interference channel gains that is observed at } \\
\text { the receive antennas. }\end{array}$ \\
\hline $\mathbf{n}_{t, i, k}$ & $\begin{array}{l}\text { Column vector of i.i.d. AWGN variants, where } \\
\text { each of the elements has zero mean and an } \\
\text { average power of } \mathbb{E}\left\{\left|n_{t, i, k, r}\right|^{2}\right\}=\sigma_{n}^{2} .\end{array}$ \\
\hline$x_{t, i, k}$ & $\begin{array}{l}\text { Transmitted data symbol from the } i \text { th transmit } \\
\text { antenna whose power is } \mathbb{E}\left\{\left|x_{t, i, k}\right|^{2}\right\}=P_{t} / N_{t} \text {. }\end{array}$ \\
\hline$x_{t, i, m}$ & $\begin{array}{l}\text { Data symbol associated with the } m \text { th } \\
\text { interference source that is by } t \text { access point } \\
\text { and affect terminal } k \text { with power } \\
\qquad \mathbb{E}\left\{\left|x_{t, i, m}\right|^{2}\right\}=P_{t, i, m} .\end{array}$ \\
\hline$g_{t, i, k, r, m}$ & Interference channel gain. \\
\hline$d_{t, k, m}$ & $\begin{array}{l}\text { Separation distance between the } m \text { th } \\
\text { interference source and the receive station } k \text {. }\end{array}$ \\
\hline $\bar{\gamma}_{D, t}$ & $\begin{array}{l}\text { Average SNR per receive antenna, and is equal } \\
\text { to } \frac{P_{t} / N_{t}}{\sigma_{n}^{2}} .\end{array}$ \\
\hline $\bar{\gamma}_{I, i, t}$ & $\begin{array}{l}\text { Average INR per interferer on the } i \text { th transmit } \\
\text { antenna, and is equal to } \frac{P_{t, i, m}}{\sigma_{n}^{2}} \text {. }\end{array}$ \\
\hline$I_{t, i}$ & Interference power per transmit antenna $i$. \\
\hline
\end{tabular}

TABLE I: Summary of main notations.

point with $t$ is used, can be written as

$$
\mathbf{z}_{t, i, k}=\mathbf{h}_{t, i, k} x_{t, i, k}+\sum_{m \in \Phi_{t}} x_{t, i, m} \mathbf{g}_{t, i, k, m}+\mathbf{n}_{t, i, k},
$$

where $\mathbf{z}_{t, i, k}=\left[z_{t, i, k, 1} z_{t, i, k, 2} \cdots z_{t, i, k, L_{k}}\right]^{T}$ is the column vector of size $L_{k} \times 1$ that contains received signal replicas at the receive array. The first term in (1) represents the received desired signal with channel vector $\mathbf{h}_{t, i, k}=\left[h_{t, i, k, 1} h_{t, i, k, 2} \cdots h_{t, i, k, L_{k}}\right]^{T}$, the second term denotes the composite PPP interference signal with channel vector $\mathbf{g}_{t, i, k, m}=$ $\left[g_{t, i, k, 1, m} g_{t, i, k, 2, m} \cdots g_{t, i, k, L_{k}, m}\right]^{T}$, and the third term de- notes the background additive white noise (AWGN) with noise vector $\mathbf{n}_{t, i, k}=\left[n_{t, i, k, 1} n_{t, i, k, 2} \cdots n_{t, i, k, L_{k}}\right]^{T}$. The interference channel gain $g_{t, i, k, r, m}$ can be expressed as $g_{t, i, k, r, m}=\alpha_{t, i, k, r, m} e^{-j \phi_{t, i, k, r, m}}\left|d_{t, k, m}\right|^{-\frac{v}{2}}$, where $\alpha_{t, i, k, r, m}$ and $\phi_{t, i, k, r, m}$ are the channel fading envelope and phase, respectively, wherein the fading envelope follows Rayleigh distribution with $\mathbb{E}\left\{\alpha_{t, i, k, r, m}^{2}\right\}=1$. The MIMO configuration implements TAS/MRC scheme and using single spectrum sub-band at a time. It is worth mentioning that there can be other design alternatives to the MIMO configuration adopted herein for which the developments in this paper can be readily applied without loss of generality. For example, with single-input multiple-output (SIMO) and the serving access point has multiple sub-bands but can serve each user through single sub-band at a time, transmit sub-band selection (TSBS) with MRC (i.e. TSBS/MRC) becomes equivalent to TAS/MRC operation. Moreover, when the desired user terminal is limited to use single receive antenna at a time and there are multiple transmit antennas (or transmit subbands that can be used simultaneously) at the serving access point, maximum ratio transmission (MRT) with adjusted transmit power per transmit antenna (or subband) along with receive antenna selection (MRT/RAS) can replace TAS/MRC design and its operation benefits. However, these systems vary in terms of complexity of operation. Here, the received signal replicas are combined via MRC, and then the transmit antenna index with the best MRC is selected at the receive station. The receive station sends back the index to the transmitter to be used for subsequent downlink packet transmission. The Pre-TAS SINR following receive MRC (after weighting $\mathbf{z}_{t, i, k}$ by $\widehat{\mathbf{h}}_{t, i, k}^{H} /\left\|\widehat{\mathbf{h}}_{t, i, k}\right\|$, where $\widehat{\mathbf{h}}_{t, i, k}=$ $\mathbf{h}_{t, i, k} /\left|d_{t, k}\right|^{-v}$ ) of the link between $t$ and $k$ when the $i$ th transmit antenna is used can be obtained as where $\sum_{r=1}^{L_{k}} \alpha_{t, i, k, r}^{2}=\widehat{\mathbf{h}}_{t, i, \mathrm{M}_{U}} \widehat{\mathbf{h}}_{t, i, \mathrm{M}_{U}}^{H}$ that is the sum of i.i.d. exponential random variables each of which has unit mean, $\widetilde{\alpha}_{t, i, k, m}^{2}=\left|\widehat{\mathbf{h}}_{t, i, k}^{H} \widehat{\mathbf{g}}_{t, i, k} /\left\|\widehat{\mathbf{h}}_{t, i, k}\right\|\right|^{2}$ follows an exponential distribution of unit mean, and $\left|\widehat{\mathbf{h}}_{t, i, k}^{H} \mathbf{n}_{t, i, k} /\left\|\widehat{\mathbf{h}}_{t, i, k}\right\|\right|^{2}$ is an AWGN distributed variant of zero mean and power $\sigma_{n}^{2}$.

By exploiting the statistical independence between small-scale fading and point processes $[18,20]$, the aggregate interference power in (2), which is denoted by $I_{t, i, k}=\sum_{m \in \Phi_{t}} \bar{\gamma}_{I, i, t}\left|d_{t, k, m}\right|^{-v} \widetilde{\alpha}_{t, i, k, m}^{2}$, has Laplace 


$$
\gamma_{\mathrm{SINR}, t, i, k}=\frac{\frac{P_{t}}{N_{t}}\left|d_{t, k}\right|^{-v}\left|\frac{\widehat{\mathbf{h}}_{t, i, k}^{H} \widehat{\mathbf{h}}_{t, i, k}}{\left\|\widehat{\mathbf{h}}_{t, i, k}\right\|}\right|^{2}}{\sum_{m \in \Phi_{t}} P_{t, i, m}\left|d_{t, k, m}\right|^{-v}\left|\frac{\widehat{\mathbf{h}}_{t, i, k}^{H} \widehat{\mathbf{g}}_{t, i, k}}{\left\|\widehat{\mathbf{h}}_{t, i, k}\right\|}\right|^{2}+\left|\frac{\widehat{\mathbf{h}}_{t, i, k}^{H} \mathbf{n}_{t, i, k}}{\left\|\mid \widehat{\mathbf{h}}_{t, i, k}\right\|}\right|^{2}}=\frac{\bar{\gamma}_{D, t}\left|d_{t, k}\right|^{-v} \sum_{r=1}^{L_{k}} \alpha_{t, i, k, r}^{2}}{\sum_{I_{t, i, k}}^{\sum_{m \in \Phi_{t}} \bar{\gamma}_{I, i, t}\left|d_{t, k, m}\right|^{-v} \widetilde{\alpha}_{t, i, k, m}^{2}+1}} .
$$

transform, $\psi_{I_{t, i, k}}(s)=\mathbb{E}\left\{e^{-s I_{t, i, k}}\right\}$, that is expressed as

$$
\begin{aligned}
\psi_{I_{t, i, k}}(s) & =\mathbb{E}\left\{\prod_{m \in \Phi_{t}} \mathbb{E}\left\{e^{-s\left(\bar{\gamma}_{I, i, t}\left|d_{t, k, m}\right|^{-v} \widetilde{\alpha}_{t, i, k, m}^{2}\right)}\right\}\right\} \\
& =\exp \left[-\lambda_{t} \int_{R^{2}}\left(1-\frac{1}{1+s \bar{\gamma}_{I, t} z^{-v}}\right) d z\right] \\
& =\exp \left[-\lambda_{t}\left(s \bar{\gamma}_{I, i, t}\right)^{\frac{2}{v}} \beta(v)\right], v>2
\end{aligned}
$$

where $\beta(v)=\frac{2 \pi^{2}}{v \sin (2 \pi / v)}$. Note that $I_{t, i, k}$ follows alpha stable distribution with characteristic exponent $\frac{2}{v}$ and dispersion $\lambda_{t}\left(\bar{\gamma}_{I, i, t}\right)^{\frac{2}{v}} \beta(v)$.

The formulation of the aggregate interference power $I_{t, i, k}$ in (3) that is observed at user $k$ may depend on the used transmit antenna (whose index is $i$ ) at the serving access point $t$. This is noticed if the transmit antennas at $t$ are separated in code domain by assigning them orthogonal codes. Note that TAS/MRC with orthogonal codes used to orthogonalize transmit antennas transmissions in code domain while using single spectrum subband is equivalent to a SIMO system with TSBS/MRC, where the sub-bands guarantee transmit orthogonality in frequency domain. In this case, interference signals observed from transmit antennas at terminal $t$ become de-correlated in code domain. On the other hand, when transmit antennas at terminal $t$ are not separated, it is reasonable to assume that $I_{t, i, k}=I_{t, k}$, for $i=1,2, \ldots, N_{t}$, which implies that interference sources observed from $t$ are identical, regardless of the used transmit antenna at terminal $t$ to serve terminal $k$. These two cases are incorporated into the findings of the following part. In addition to these two cases of orthogonal and identical $\left\{I_{t, i, k}\right\}_{i=1}^{N_{t}}$, correlated $\left\{I_{t, i, k}\right\}_{i=1}^{N_{t}}$ may be observed when transmit antennas are not perfectly orthogonal in code domain (note this correlation is practically infeasible in SIMO systems that uses multiple sub-bands to either implement MRT/RAS or TSBS/MRC that use guard sub-bands in frequency domain). This can be captured via the cross-correlation between $\left\{I_{t, i, k}\right\}_{i=1}^{N_{t}}$ underlying small-scale fading powers $\left\{\widetilde{\alpha}_{t, i, k, m}^{2}\right\}$. Given that these terms are results of complex-valued Gaussian models of interference small-scale fading channel gains, correlation models of underlaying complex-valued Gaussian processes can be incorporated into the analysis of the adopted TAS scheme, wherein related results in [21] for selection combining can be useful. The expected effect of correlation among $\left\{I_{t, i, k}\right\}_{i=1}^{N_{t}}$ under TAS scheme is bounded between those of the orthogonal and identical $\left\{I_{t, i, k}\right\}_{i=1}^{N_{t}}$. Further details on this case are not shown herein, but may be reported in a future work.

\section{TAS Scenarios}

The selection of the suitable transmit antenna at the serving station $t$ can be implemented to either maximize the desired combined SNR after MRC (i.e., SNR-based TAS) at the receive station or maximize the Pre-TAS SINR (i.e., combined SINR prior to TAS) in (2). The SNR-based TAS is apparently less complex than the SINR-based TAS since it does not require the knowledge of interference power in the identification of the suitable transmit antenna index. Moreover, the SNRbased selection will provide identical performance to that of the SINR-based selection when interference power on transmit antennas are identical (i.e., the case of $I_{t, i, k}=I_{t, k}$, for $i=1,2, \ldots, N_{t}$, as discussed above).

1) SNR-Based Post-TAS: For the case when the transmit antennas are perfectly orthogonal, it follows that the SNR-based TAS results in the Post-TAS SINR (i.e., the combined SINR after TAS), $\gamma_{\mathrm{SINR}, t, k}$, that is given

$$
\gamma_{\mathrm{SINR}, t, k}=\frac{\max _{i=1, \ldots, N_{t}}\left\{\gamma_{\mathrm{SNR}, t, i, k}\right\}}{I_{t, i^{\star}, k}+1},
$$

where $\gamma_{\mathrm{SNR}, t, i, k}=\bar{\gamma}_{D, t}^{\prime} \sum_{r=1}^{L_{k}} \alpha_{t, i, k, r}^{2}$ is the combined Pre-TAS SNR, $\bar{\gamma}_{D, t}^{\prime}=\bar{\gamma}_{D, t}\left\|d_{t, k}\right\|^{-v}$, and $I_{t, i^{\star}, k}=I_{t, i, k}$ if the $i$ th transmit antenna is selected. On the other hand, when the interference powers on transmit antennas are identical, it follows that $\gamma_{\mathrm{SINR}, t, k}$ has the same form in (4) but with $I_{t, i^{\star}, k}=I_{t, k}$, for any $i=1, \ldots, N_{t}$.

2) SINR-Based Post-TAS: For the case when the transmit antennas are perfectly orthogonal, it follows that the SINR-based TAS results in the Post-TAS SINR, $\widetilde{\gamma}_{\mathrm{SINR}, t, k}$, that is given

$$
\widetilde{\gamma}_{\mathrm{SINR}, t, k}=\max _{i=1, \ldots, N_{t}}\left\{\frac{\gamma_{\mathrm{SNR}, t, i, k}}{I_{t, i, k}+1}\right\} .
$$

On the other hand, when the interference powers on transmit antennas are identical, it follows that $\gamma_{\mathrm{SINR}, t, k}$ has the same formulation in (4) with $I_{t, i^{\star}, k}=I_{t, k}$, for any $i=1, \ldots, N_{t}$. 


\section{DESCRIPTION OF HyBRID CONTROL-ACCESS SCHEME}

For the sake of simplicity, the following analysis considers macrocell user (i.e., $k=\mathrm{M}_{U}$ ) as being the user of interest. However, in light of the preceding results in the previous section, the analysis can be modified directly to treat femtocell user $k=\mathrm{F}_{U}$. Now for the macrocell user $\mathrm{M}_{U}$, the selection of the suitable downlink (i.e., the macrocell base station or a femtocell access point) to serve $\mathrm{M}_{U}$ can be made according to different algorithms. The following analysis outlines some of these algorithms and explain their applicability:

\section{A. Post-TAS Hybrid Access}

The selection of the suitable access point can be related to the Post-TAS SINRs levels observed at macrocell user $\mathrm{M}_{U}$. The following discussion considers the SNRbased and SINR-based Post-TAS schemes discussed in subsections II-C1 and II-C2, respectively.

1) SNR-Based Post-TAS Hybrid Access: Based on the results in subsection II-C1, the ratios of the resulting SINRs at $\mathrm{M}_{U}$ can be expressed as

$$
\begin{aligned}
\rho_{\mathrm{SINR}} & =\eta_{0} \frac{\gamma_{\mathrm{SINR}, F_{B}, M_{U}}}{\gamma_{\mathrm{SINR}, M_{B}, M_{U}}}, \\
& =\eta_{0} \frac{\max _{i=1, \ldots, N_{F_{B}}}\left\{\gamma_{\mathrm{SNR}, F_{B}, i, M_{U}}\right\}}{\max _{i=1, \ldots, N_{M_{B}}}\left\{\gamma_{\mathrm{SNR}, M_{B}, i, M_{U}}\right\}} \frac{I_{M_{B}, i^{\star}, M_{U}}+1}{I_{F_{B}, i^{\star}, M_{U}}+1},
\end{aligned}
$$

where $I_{M_{B}, i^{\star}, M_{U}}$ and $I_{F_{B}, i^{\star}, M_{U}}$ are statistically independent aggregate interference powers that are observed at the macrocell user $M_{U}$, and they originated due to the service provided to other concurrently active users by spatially independent macrocell $M_{B}$ and a nearby femtocell $F_{B}$ access points. The number of femtocell access points $\Phi_{\mathrm{F}_{B}}(A)$ in close vicinity of $\mathrm{M}_{U}$ over an area $A$ follows a Poisson distribution with mean value of $\lambda_{\mathrm{F}_{B}} A$, and number of femtocell users $\Phi_{\mathrm{F}_{U}}(A)$ in the same area follows also a Poisson distribution with mean value of $\lambda_{\mathrm{F}_{U}} A$. The condition to allow for a hybrid access for $\mathrm{M}_{U}$ is given by the likelihood that the femtocell access points are capable to serve all femtocell users in area $A$ and have free resources to support a service to $\mathrm{M}_{U}$, which gives the event that $\Phi_{\mathrm{F}_{U}}(A)<\Phi_{\mathrm{F}_{B}}(A)$. Then it follows that

$$
\begin{aligned}
\eta_{0} & =\operatorname{Pr}\left\{\frac{\Phi_{\mathrm{F}_{U}}(A)}{\Phi_{\mathrm{F}_{B}}(A)}<1\right\} \\
& =\sum_{x=0}^{\infty}\left[\sum_{n=0}^{x-1} e^{-\lambda_{\mathrm{F}_{U}} A} \frac{\left(\lambda_{\mathrm{F}_{U}} A\right)^{n}}{n !}\right] e^{-\lambda_{\mathrm{F}_{B}} A} \frac{\left(\lambda_{\mathrm{F}_{B}} A\right)^{x}}{x !},
\end{aligned}
$$

which indicates the likelihood of observing at least one free femtocell access point over $A$ that can serve $\mathrm{M}_{U}$.
If $\rho_{\text {SINR }}<1$, then $\mathrm{M}_{U}$ will be served by the macrocell base station $\mathrm{M}_{B}$. Otherwise, $\mathrm{M}_{U}$ will be served by a femtocell access point $\mathrm{F}_{B}$. The probability of the event $\rho_{\text {SINR }}<1$ can be expressed as

$$
\begin{aligned}
& \operatorname{Pr}\left\{\rho_{\mathrm{SINR}}<1\right\} \\
& =\int_{0}^{+\infty} F_{\gamma_{\mathrm{SINR}, F_{B}, M_{U}}}\left(\frac{x}{\eta_{0}}\right) f_{\gamma_{\mathrm{SINR}, M_{B}, M_{U}}}(x) d x,
\end{aligned}
$$

where $F_{\gamma_{\mathrm{SINR}, F_{B}, M_{U}}}(y)$ and $f_{\gamma_{\mathrm{SINR}, M_{B}, M_{U}}}(y)$ can be deduced as will be explained in Section IV.

The preceding algorithm can result in frequent handover of macrocell user $M_{U}$ due to the rapid variations of the small-scale fading conditions in the resulting SNR. To reduce the rate of handover association, a more robust algorithm can be implemented based on largescale fading with small-scale fading associated with the Post-TAS desired SNR be averaged out. Consequently, the result in (6) reduces to

$$
\bar{\rho}_{\mathrm{SINR}}=\bar{\eta}_{0} \frac{I_{M_{B}, i^{\star}, M_{U}}+1}{I_{F_{B}, i^{\star}, M_{U}}+1} \approx \bar{\eta}_{0} \frac{I_{M_{B}, i^{\star}, M_{U}}}{I_{F_{B}, i^{\star}, M_{U}}},
$$

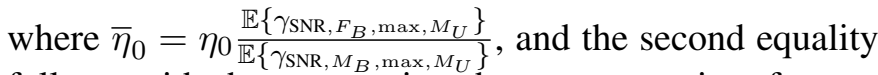
follows with the assumption that aggregate interference power are relatively large quantities. The result in (9) reveals that the access point association will be mainly based on the large-scale statistics of aggregate Poisson field interference models. Then, the likelihood of association event that $\bar{\rho}_{\text {SINR }}<1$ can be expressed as

$$
\begin{aligned}
& \operatorname{Pr}\left\{\bar{\rho}_{\mathrm{SINR}}<1\right\} \\
& =\int_{0}^{+\infty} F_{I_{M_{B}, i^{\star}, M_{U}}}\left(\frac{x+1}{\bar{\eta}_{0}}-1\right) f_{I_{F_{B}, i^{\star}, M_{U}}}(x) d x \\
& \approx \int_{0}^{+\infty} F_{I_{M_{B}, i^{\star}, M_{U}}}\left(\frac{x}{\bar{\eta}_{0}}\right) f_{I_{F_{B}, i^{\star}, M_{U}}}(x) d x
\end{aligned}
$$

where $F_{I_{F_{B}, i^{\star}, M_{U}}}(y)$ and $f_{I_{M_{B}, i^{\star}, M_{U}}}(y)$ can be deduced from the statistical aggregate interference models in (3), in which the index $i^{\star}$ denotes the index of the selected transmit antenna on the macrocell or femtocell link. Note again that the knowledge of $i^{\star}$ becomes unnecessary and has no effect under identical interference on transmit channels.

2) SINR-Based Post-TAS Hybrid Access: Based on the results in subsection II-C2, the ratios of the resulting SINRs at $\mathrm{M}_{U}$ can be expressed as

$\widetilde{\rho}_{\mathrm{SINR}}=\frac{\widetilde{\gamma}_{\mathrm{SINR}, F_{B}, M_{U}}}{\widetilde{\gamma}_{\mathrm{SINR}, M_{B}, M_{U}}}=\frac{\max _{i=1, \ldots, N_{F_{B}}}\left\{\frac{\gamma_{\mathrm{SNR}, F_{B}, i, M_{U}}}{I_{F_{B}, i, M_{U}}+1}\right\}}{\max _{i=1, \ldots, N_{M_{B}}}\left\{\frac{\gamma_{\mathrm{SNR}, M_{B}, i, M_{U}}}{I_{M_{B}, i, M_{U}}+1}\right\}}$. 
The probability of the event $\widetilde{\rho}_{\text {SINR }}<1$ can be expressed as

$$
\begin{aligned}
& \operatorname{Pr}\left\{\widetilde{\rho}_{\mathrm{SINR}}<1\right\} \\
& =\int_{0}^{+\infty} F_{\widetilde{\gamma}_{\mathrm{SINR}, F_{B}, M_{U}}}\left(\frac{x}{\eta_{0}}\right) f_{\widetilde{\gamma}_{\mathrm{SINR}, M_{B}, M_{U}}}(x) d x,
\end{aligned}
$$

where $F_{\widetilde{\gamma}_{\mathrm{SINR}, F_{B}, M_{U}}}(y)$ and $f_{\widetilde{\gamma}_{\mathrm{SINR}, M_{B}, M_{U}}}(y)$ can be deduced as will be explained in Section VII. For the case when the interference powers on transmit antennas are identical (i.e., $I_{t, i^{\star}, k}=I_{t, k}$, for any $i=1, \ldots, N_{t}$ ), the result in (11) reduces to a form that is similar to (6) without the inclusion of the index $i^{\star}$ of the selected transmit antenna. Consequently, the findings in (9) and (12) are applicable under identical interference on transmit antennas.

\section{B. Pre-TAS Hybrid Access: Interference-Aware Scenario}

For the case when the hybrid access scheme is performed ahead of TAS scheme, which realizes Pre-TAS hybrid access, the algorithms described in subsection III-A1 cannot be adopted. In the absence of information about the selected transmit antenna, and to maintain a robust macrocell user association with reduced handover rate, the findings in (9) can be extended for an interferenceware Pre-TAS hybrid access scheme. Since the transmit antenna that meets the Post-TAS SINR requirement is still unknown, and hence, the aggregate interference that is associated with that transmit antenna is unpredictable, a practical solution can be through considering the aggregate interference power on all transmit antennas at each access point, which give $I_{\mathrm{M}_{B}}=\sum_{i=1}^{N_{\mathrm{M}_{B}}} I_{\mathrm{M}_{B}, i, \mathrm{M}_{U}}$ and $I_{\mathrm{F}_{B}}=\sum_{i=1}^{N_{\mathrm{F}_{B}}} I_{\mathrm{F}_{B}, i, \mathrm{M}_{U}}$. Therefore, the association parameter in this case can be defined as

$$
\rho=\eta_{0} \frac{I_{\mathrm{M}_{B}}+1}{I_{\mathrm{F}_{B}}+1} \approx \eta_{0} \frac{I_{\mathrm{M}_{B}}}{I_{\mathrm{F}_{B}}} .
$$

which is a variant of that adopted in [22] as a hybrid access association parameter.

It is reasonable to consider the cases when the aggregate interference power observed at $\mathrm{M}_{U}$ is identical on all transmit antennas or un-correlated among all transmit antennas of the serving access point, as discussed in the preceding part, and revisited below.

1) Scenario A of interference: This scenario A of interference refers to the case when $\mathrm{M}_{U}$ undergoes the same interference effect from all transmit antennas of its serving access point. Therefore, $I_{\mathrm{M}_{B}}=N_{M_{B}} I_{\mathrm{M}_{B}, M_{U}}$ and $I_{\mathrm{F}_{B}}=N_{F_{B}} I_{\mathrm{F}_{B}, M_{U}}$, regardless of the selected transmit antenna index. Hence, $\rho$ in (13) is redefined as $\rho_{1} \approx \eta \frac{I_{\mathrm{M}_{B}, M_{U}}}{I_{\mathrm{F}_{B}, M_{U}}}$, where $\eta=\eta_{0} N_{M_{B}} / N_{F_{B}}$. The following proposition provides the likelihood of the association event that $\rho_{1}<1$.
Proposition 1: The term $\operatorname{Pr}\left\{\rho_{1}<1\right\}$ can be expressed as

$$
\begin{aligned}
\operatorname{Pr}\left\{\rho_{1}<1\right\} & =\int_{0}^{+\infty} F_{I_{\mathrm{M}_{B}}}\left(\frac{x}{\eta}\right) f_{I_{\mathrm{F}_{B}}}(x) d x, \\
& =\frac{v}{2} H_{2,2}^{1,1}\left[\left.\frac{\lambda_{\mathrm{M}_{B}}}{\lambda_{\mathrm{F}_{B}}}\left(\frac{\bar{\gamma}_{I, \mathrm{M}_{B}}}{\eta \bar{\gamma}_{I, \mathrm{~F}_{B}}}\right)^{\frac{2}{v}}\right|_{(0,1),\left(1, \frac{2}{v}\right)} ^{(1,1),\left(1, \frac{2}{v}\right)}\right] .
\end{aligned}
$$

Proof: See Appendix A.

2) Scenario $B$ of Interference: This scenario happens when $\mathrm{M}_{U}$ undergoes independent interference on transmit antennas. Therefore, the likelihood of the association event $\rho<1$, which is redefined as $\rho_{2}<1$ for simplicity, is provided below.

Proposition 2: The term $\operatorname{Pr}\left\{\rho_{2}<1\right\}$ can be obtained as

$$
\begin{aligned}
& \operatorname{Pr}\left\{\rho_{2}<1\right\} \\
& =\frac{v}{2} H_{2,2}^{1,1}\left[\left.\frac{\lambda_{\mathrm{M}_{B}}}{\lambda_{\mathrm{F}_{B}}} \frac{\sum_{i=1}^{N_{\mathrm{M}_{B}}}\left(\bar{\gamma}_{I, \mathrm{M}_{B}, i}\right)^{\frac{2}{v}}}{\left(\eta_{0}\right)^{\frac{2}{v}} \sum_{i=1}^{N_{\mathrm{F}_{B}}}\left(\bar{\gamma}_{I, \mathrm{~F}_{B}, i}\right)^{\frac{2}{v}}}\right|_{(0,1),\left(1, \frac{2}{v}\right)} ^{(1,1),\left(1, \frac{2}{v}\right)}\right] .
\end{aligned}
$$

Proof: See Appendix B.

It is worth mentioning that $\operatorname{Pr}\left\{\rho_{2}<1\right\}$ in (15) becomes identical to $\operatorname{Pr}\left\{\rho_{1}<1\right\}$ in (14) when $\bar{\gamma}_{I, t, i}=\bar{\gamma}_{I, t}$, for $i=1,2, \ldots, N_{t}$ and $t=\left\{M_{B}, F_{B}\right\}$.

The hybrid access algorithms described above in subsections III-A and III-B can be readily adopted for the remaining parts of this paper. For instance, the results developed in subsection III-A considering Pre-TAS interference-aware hybrid access scheme will be used in the remaining Sections to provide generalized results that can applied for different conditions of interference on transmit antennas at the serving access point.

\section{Performance Analysis Using SNR-Based Post-TAS}

This Section presents exact results for some performance measures of the SNR-based Post-TAS presented in subsection II-C1, considering the hybrid access schemes in subsection III-B. The results are presented in two parts for the outage probability and average error rate of the desired macrocell user $\mathrm{M}_{U}$.

\section{A. Exact Outage Probability}

The outage probability is defined as the probability that the combined SINR at $\mathrm{M}_{U}$ falls below a predetermined threshold, $\gamma_{t h} ; \mathrm{P}_{\text {out }}=\operatorname{Pr}\left\{\gamma_{\text {SINR }}<\gamma_{t h}\right\}$. The following parts present the outage probability for the two cases of interference on transmit antennas based on the findings in (14) and (15). 
1) Scenario A of Interference: The outage probability in this case can be expressed as

$$
\begin{aligned}
\mathrm{P}_{\mathrm{out}} & =\operatorname{Pr}\left\{\rho_{1}<1\right\} \operatorname{Pr}\left\{\gamma_{\mathrm{SINR}, \mathrm{M}_{B}, \mathrm{M}_{U}}<\gamma_{t h}\right\} \\
& +\left(1-\operatorname{Pr}\left\{\rho_{1}<1\right\}\right) \operatorname{Pr}\left\{\gamma_{\mathrm{SINR}, \mathrm{F}_{B}, \mathrm{M}_{U}}<\gamma_{t h}\right\},
\end{aligned}
$$

where $\operatorname{Pr}\left\{\rho_{1}<1\right\}$ is given in (14) and $\gamma_{\mathrm{SINR}, t, k}$ is defined in (4) with $I_{t, k}=I_{t, i, k}$, regardless of the index of the selected transmit antenna.

Proposition 3: The term $\operatorname{Pr}\left\{\gamma_{\mathrm{SINR}, t, k}<\gamma_{t h}\right\}$ in (16) for $t=\left\{\mathrm{M}_{B}, \mathrm{~F}_{B}\right\}$ and $k=\mathrm{M}_{U}$ is expressed as in (17)

Proof: See Appendix $C$.

Substituting the resulting expression of $\operatorname{Pr}\left\{\gamma_{\mathrm{SINR}, t, k}<\right.$ $\left.\gamma_{t h}\right\}$ for $t=\left\{\mathrm{M}_{B}, \mathrm{~F}_{B}\right\}$ and $k=\mathrm{M}_{U}$ in (16) yields the desired final results for the outage performance of $\mathrm{M}_{U}$. 2) Scenario B of Interference: The outage probability in this case can be expressed as

$$
\begin{aligned}
\mathrm{P}_{\text {out }} & =\operatorname{Pr}\left\{\rho_{2}<1\right\} \operatorname{Pr}\left\{\gamma_{\mathrm{SINR}_{\mathrm{M}} \mathrm{M}_{B}, \mathrm{M}_{U}}<\gamma_{t h}\right\} \\
& +\left(1-\operatorname{Pr}\left\{\rho_{2}<1\right\}\right) \operatorname{Pr}\left\{\gamma_{\mathrm{SINR}, \mathrm{F}_{B}, \mathrm{M}_{U}}<\gamma_{t h}\right\},
\end{aligned}
$$

where $\operatorname{Pr}\left\{\rho_{2}<1\right\}$ is given in (15) and $\gamma_{\mathrm{SINR}, t, k}$ is defined in (4) with $I_{t, k}=I_{t, i, k}$ only if the $i$ th transmit antenna is selected.

Proposition 4: The term $\operatorname{Pr}\left\{\gamma_{\mathrm{SINR}, t, k}<\gamma_{t h}\right\}$ in (18) for $t=\left\{\mathrm{M}_{B}, \mathrm{~F}_{B}\right\}$ and $k=\mathrm{M}_{U}$ is as in (19) Proof: See Appendix $D$.

Substituting the resulting expression of $\operatorname{Pr}\left\{\gamma_{\mathrm{SINR}, t, k}<\right.$ $\left.\gamma_{t h}\right\}$ for $t=\left\{\mathrm{M}_{B}, \mathrm{~F}_{B}\right\}$ and $k=\mathrm{M}_{U}$ in (18) yields the desired final results for the outage performance of $\mathrm{M}_{U}$.

\section{B. Exact Average Error Rate}

The following parts address the average error rate performance of $\mathrm{M}_{U}$.

1) Scenario A of Interference: The average error rate in this case can be defined as

$$
\begin{aligned}
\mathrm{Pe} & =\operatorname{Pr}\left\{\rho_{1}<1\right\} \mathrm{Pe}_{\mathrm{M}_{B}, \mathrm{M}_{U}} \\
& +\left(1-\operatorname{Pr}\left\{\rho_{1}<1\right\}\right) \mathrm{Pe}_{\mathrm{F}_{B}, \mathrm{M}_{U}},
\end{aligned}
$$

The average error rate $\mathrm{Pe}_{t, k}$ in (20) for $t=\left\{\mathrm{M}_{B}, \mathrm{~F}_{B}\right\}$ and $k=\mathrm{M}_{U}$ can be expressed as [26]

$$
\mathrm{Pe}_{t, k}=\frac{1}{\pi} \int_{0}^{\pi(M-1) / M} \mathcal{M}_{\gamma_{\mathrm{SINR}, t, k}}\left(\frac{\sin ^{2}(\pi / M)}{\sin ^{2} \theta}\right) d \theta,
$$

where $M$ is the constellation size. According to [27], the integral can be avoided by the using approximation as in (22) Using the MGF definition $\mathcal{M}_{\gamma_{\mathrm{SINR}, t, k}}(s)=s \int_{0}^{\infty} e^{-s \gamma_{t h}} \operatorname{Pr}\left\{\gamma_{\mathrm{SINR}, t, k}<\gamma_{t h}\right\} d \gamma_{t h}$, and the Fox $\mathrm{H}$-function property in [23, eq. 6], $\mathcal{M}_{\gamma_{\mathrm{SINR}, t, k}}(s)$ is obtained as in (23) where $\triangle_{t}=$

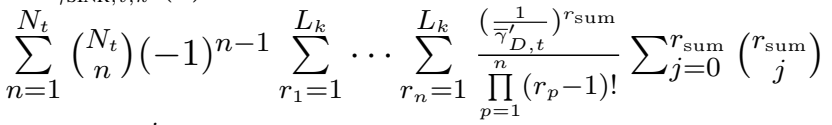
$\left(-\frac{\bar{\gamma}_{D, t}^{\prime}}{n}\right)^{j}$. Inserting the expression in (23) into gives the expression of $\mathrm{Pe}_{t, k}$, and then using the this result in (20) for $t=\left\{\mathrm{M}_{B}, \mathrm{~F}_{B}\right\}$ and $k=\mathrm{M}_{U}$ yields the desired final results for the average error rate performance of $\mathrm{M}_{U}$.

2) Scenario B of Interference: The average error rate in this case can be expressed as

$$
\begin{aligned}
\mathrm{Pe} & =\operatorname{Pr}\left\{\rho_{2}<1\right\} \mathrm{Pe}_{\mathrm{M}_{B}, \mathrm{M}_{U}} \\
& +\left(1-\operatorname{Pr}\left\{\rho_{2}<1\right\}\right) \mathrm{Pe}_{\mathrm{F}_{B}, \mathrm{M}_{U}},
\end{aligned}
$$

where $\operatorname{Pr}\left\{\rho_{2}<1\right\}$ is given in (15). Herein, $\gamma_{\mathrm{SINR}, t, k}$ is defined in (4) with $I_{t, k}=I_{t, i, k}$ only if the $i$ th transmit antenna is selected. Similarly, the term $\mathcal{M}_{\gamma_{\mathrm{SINR}, t, k}}(s)$ has the same expression as in (23) but after replacing $\alpha_{t}$ by $\phi_{t}$. Substituting the resulting expression of $\mathrm{Pe}_{t, k}$ for $t=\left\{\mathrm{M}_{B}, \mathrm{~F}_{B}\right\}$ and $k=\mathrm{M}_{U}$ into (24) yields the desired final results for the average error rate performance of $\mathrm{M}_{U}$.

\section{Asymptotic Results with SNR-BAsed Post-TAS}

This Section provides asymptotic results for the outage probability and average error rate obtained in the preceding Section in high SNR regime.

\section{A. Asymptotic Outage Probability}

The results herein are obtained using the Taylor series expansion of exponentials $\left(e^{-x} \approx 1-x\right)$ as $x \rightarrow 0$ [28, eq.1.211.1] and the asymptotic expansion of $\mathrm{H}$-function at $z=0$ [19, eq.1.8.1].

1) Scenario A of Interference: The asymptotic outage probability in (17) can be expressed as

$$
\begin{aligned}
\mathrm{P}_{t, k}^{\infty} & =\lim _{\bar{\gamma}_{D, t}^{\prime} \rightarrow \infty} \operatorname{Pr}\left\{\gamma_{\mathrm{SINR}, t, k}<\gamma_{t h}\right\}, \\
& =\left(G_{c} \bar{\gamma}_{D, t}^{\prime}\right)^{-G_{d}}+o\left(\bar{\gamma}_{D, t}^{\prime}-G_{d}\right), \\
& \approx \frac{\alpha_{t}}{\left(L_{k} !\right)^{N_{t}}}\left(\frac{\gamma_{t h}}{\bar{\gamma}_{D, t}^{\prime}}\right)^{\min \left(\frac{2}{v}, L_{k} N_{t}\right)},
\end{aligned}
$$

where $o($.$) represents f(x)=o(g(x))$ as $x \rightarrow x_{0}$ if $\lim _{x \rightarrow x_{0}} \frac{f(x)}{g(x)}=0, G_{d}$ is the diversity gain and is defined as the slope of the asymptotic curve which is given by $G_{d}=\lim _{\bar{\gamma}_{D, t}^{\prime} \rightarrow \infty} \frac{-\log \operatorname{Pr}\left\{\gamma_{\mathrm{SINR}, t, k}<\gamma_{t h}\right\}}{\log \bar{\gamma}_{D, t}^{\prime}}$, and $G_{c}$ is the coding gain representing the SNR advantage of the asymptotic curve relative to $\left(\bar{\gamma}_{D, t}^{\prime}\right)^{-G_{c}}$ reference. Comparing the results in (25) with those and (26), the achievable diversity and coding gain can be expressed as follows: $G_{d}^{\mathrm{Id}}=\min \left(\frac{2}{v}, L_{k} N_{t}\right)$ and $G_{c}^{\mathrm{Id}}=$ $\left(\frac{\alpha_{t}}{\bar{\gamma}_{D, t}^{\prime}\left(L_{k} !\right)^{N_{t}}}\right)^{-\frac{1}{\min \left(\frac{2}{v}, L_{k} N_{t}\right)}}$. This concludes that the outage probability performance is dominated by the effect of the path loss of the interference sources. Substituting the resulting $\mathrm{P}_{t, k}^{\infty}$ in (26) for $t=\left\{\mathrm{M}_{B}, \mathrm{~F}_{B}\right\}$ and $k=\mathrm{M}_{U}$ in 
$\operatorname{Pr}\left\{\gamma_{\mathrm{SINR}, t, k}<\gamma_{t h}\right\}=1-\sum_{n=1}^{N_{t}}\left(\begin{array}{c}N_{t} \\ n\end{array}\right)(-1)^{n-1} e^{-\left(n \gamma_{t h} / \bar{\gamma}_{D, t}^{\prime}\right)} \sum_{r_{1}=1}^{L_{k}} \cdots \sum_{r_{n}=1}^{L_{k}} \frac{\left(\frac{\gamma_{\text {th }}}{\bar{\gamma}_{D, t}^{\prime}}\right)^{r_{\mathrm{sum}}}}{\prod_{p=1}^{n}\left(r_{p}-1\right) !} \sum_{j=0}^{r_{\mathrm{sum}}}\left(\begin{array}{c}r_{\mathrm{sum}} \\ j\end{array}\right) \mathcal{J}_{1}$

$\operatorname{Pr}\left\{\gamma_{\mathrm{SINR}, t, k}<\gamma_{t h}\right\}=1-\sum_{n=1}^{N_{t}}\left(\begin{array}{c}N_{t} \\ n\end{array}\right)(-1)^{n-1} e^{-\left(n \gamma_{t h} / \bar{\gamma}_{D, t}^{\prime}\right)} \sum_{r_{1}=1}^{L_{k}} \cdots \sum_{r_{n}=1}^{L_{k}} \frac{\left(\frac{\gamma_{t h}}{\bar{\gamma}_{D, t}^{\prime}}\right)^{r_{\text {sum }}}}{\prod_{p=1}^{n}\left(r_{p}-1\right) !} \sum_{j=0}^{r_{\text {sum }}}\left(\begin{array}{c}r_{\text {sum }} \\ j\end{array}\right) \mathcal{J}_{3}$

$$
\begin{aligned}
\mathrm{Pe}_{t, k} \approx & \left(\frac{M-1}{2 \pi M}-\frac{1}{6}\right) \mathcal{M}_{\gamma_{\mathrm{SINR}, t, k}}\left(-\sin ^{2}(\pi / M)\right)+\frac{1}{4} \mathcal{M}_{\gamma_{\mathrm{SINR}, t, k}}\left(\frac{-4 \sin ^{2}(\pi / M)}{3}\right)+\left(\frac{M-1}{2 \pi M}-\frac{1}{4}\right) \\
& \times \mathcal{M}_{\gamma_{\mathrm{SINR}, t, k}}\left(\frac{\sin ^{2}(\pi / M)}{\sin ^{2} \theta}\right) .
\end{aligned}
$$

$$
\mathcal{M}_{\gamma_{\mathrm{SIN}, t, k}}(s)=1-s \triangle_{t}\left(s+\frac{n}{\bar{\gamma}_{D, t}^{\prime}}\right)^{j-r_{\mathrm{sum}}-1} H_{2,2}^{2,1}\left[\left.\alpha_{t}\left(\frac{n}{\bar{\gamma}_{D, t}^{\prime}}\right)^{\frac{2}{v}}\left(s+\frac{n}{\bar{\gamma}_{D, t}^{\prime}}\right)^{-\frac{2}{v}}\right|_{\left(j, \frac{2}{v}\right),(0,1)} ^{\left(j-r_{\mathrm{sum}}, \frac{2}{v}\right),\left(0, \frac{2}{v}\right)}\right],
$$

(16) yields the desired the asymptotic outage probability of $\mathrm{M}_{U}$.

2) Scenario B of Interference: Using (17) and replacing $\mathcal{J}_{1}$ by $\mathcal{J}_{3}$, the asymptotic outage probability can be obtained as

$$
\mathrm{P}_{t, k}^{\infty} \approx \frac{\phi_{t}}{\left(L_{k} !\right)^{N_{t}}}\left(\frac{\gamma_{t h}}{\bar{\gamma}_{D, t}^{\prime}}\right)^{\min \left(\frac{2}{v}, L_{k} N_{t}\right)}
$$

Now, the associated diversity and coding gains can be obtained as follows: $G_{d}^{\text {Ind }}=\min \left(\frac{2}{v}, L_{k} N_{t}\right)$ and $G_{c}^{\text {Ind }}=\left(\frac{\phi_{t}}{\bar{\gamma}_{D, t}^{\prime}\left(L_{k} !\right)^{N_{t}}}\right)^{-\frac{1}{\min \left(\frac{2}{v}, L_{k} N_{t}\right)}}$. Substituting the resulting expression of $\mathrm{P}_{t, k}^{\infty}$ for $t=\left\{\mathrm{M}_{B}, \mathrm{~F}_{B}\right\}$ and $k=\mathrm{M}_{U}$ in (18) yields the desired asymptotic outage probability performance of $\mathrm{M}_{U}$. The outage performance of this scenario is also dominated by the pass loss of the interference sources.

As can be observed from (26) and (27), the considered system under identical and independent interference scenarios has the same diversity gain, but the SINR gap between the two scenarios depend on the coding gain. This SINR gap is characterised as $\frac{G_{c}^{\text {Id }}}{G_{c}^{\operatorname{Ind}}}=\left(\frac{\alpha_{t}}{\phi_{t}}\right)^{-\frac{1}{\min \left(\frac{2}{v}, L_{k} N_{t}\right)}}$, which indicates that, for the same outage probability, the performance under identical interference outperforms that of independent interference on same transmit antennas by $10\left(1 / \min \left(\frac{2}{v}, L_{k} N_{t}\right)\right) \log _{10}\left(\alpha_{t} / \phi_{t}\right) \mathrm{dB}$.

\section{B. Asymptotic Average Error Rate}

1) Scenario A of Interference: The asymptotic MGF, $\mathcal{M}_{t, k}^{\infty}(s)$, can be obtained as in (28). Thus, inserting the MGF obtained into (21), the average error rate is expressed as

$$
\begin{aligned}
\mathrm{Pe}_{t, k}^{\infty} & \approx \frac{\Gamma\left(\min \left(\frac{2}{v}, L_{k} N_{t}\right)+1\right)}{\pi\left(L_{k} !\right)^{N_{t}}} \alpha_{t} \\
& \times\left(\frac{1}{\bar{\gamma}_{D, t}^{\prime} \sin ^{2}(\pi / 2)}\right)^{\min \left(\frac{2}{v}, L_{k} N_{t}\right)} \mathcal{I}_{1},
\end{aligned}
$$

where $\mathcal{I}_{1}$ can be re-expressed, after utilizing the periodicity of sine function, as

$$
\begin{aligned}
\mathcal{I}_{1} & =\int_{0}^{\pi / 2}\left(\sin ^{2} \theta\right)^{g} d \theta+\int_{\pi / M}^{\pi / 2}\left(\sin ^{2} \theta\right)^{g} d \theta \\
& =\frac{\sqrt{\pi} \Gamma(0.5+g)}{2 \Gamma(1+g)}+\sqrt{1-\sin ^{2}(\pi / M)} \\
& \times{ }_{2} F_{1}\left(0.5,0.5-g ; 1.5 ; 1-\sin ^{2}(\pi / M)\right) .
\end{aligned}
$$

Inserting the resulting expression of $\mathrm{Pe}_{t, k}^{\infty}$ for $t=$ $\left\{\mathrm{M}_{B}, \mathrm{~F}_{B}\right\}$ and $k=\mathrm{M}_{U}$ into (20) yields the desired asymptotic average error rate of $\mathrm{M}_{U}$.

2) Scenario B of Interference: The expression for $\mathrm{Pe}_{t, k}^{\infty}$ in this case is similar to that in (29) after replacing $\alpha_{t}$ by $\phi_{t}$. Inserting this resulting $\mathrm{Pe}_{t, k}^{\infty}$ for $t=\left\{\mathrm{M}_{B}, \mathrm{~F}_{B}\right\}$ and $k=\mathrm{M}_{U}$ into (24) yields the desired result.

\section{EFFect of FeEdback Delay and ARbitrary TAS PROCESSING}

This Section analyzes the performance of the SNR-Based Post-TAS under imperfect channel feedback, which can be due to noisy channel estimation, feedback delay, or channel quantization error. In specific, the analysis considers the feedback delay effect, and the TAS scheme 


$$
\mathcal{M}_{t, k}^{\infty}(s) \approx \frac{\alpha_{t}}{\left(L_{k} !\right)^{N_{t}}} s^{-\min \left(\frac{2}{v}, L_{k} N_{t}\right)} \Gamma\left(\min \left(\frac{2}{v}, L_{k} N_{t}\right)+1\right)\left(\frac{1}{\bar{\gamma}_{D, t}^{\prime}}\right)^{\min \left(\frac{2}{v}, L_{k} N_{t}\right)}
$$

is generalized for an arbitrary TAS from the associated order statistics.

The suitable transmit antenna is chosen based on the estimated channel, and this requires full synchronization between the serving station and the user of interest $\mathrm{M}_{U}$ with perfect feedback. However, due to channel impairments, there might be a time lag $\tau_{d_{t, k}}$ between the selection of the transmit antenna and its time of use. To capture this effect, let the true channel gain be $\mathbf{h}_{t, k}$ and its delayed version be $\tilde{\mathbf{h}}_{t, k}$. The relation between $\mathbf{h}_{t, k}$ and $\tilde{\mathbf{h}}_{t, k}$ is expressed as $\tilde{\mathbf{h}}_{t, k}=\sqrt{\rho_{\tau_{t, k}}} \mathbf{h}_{t, k}+\sqrt{1-\rho_{\tau_{t, k}}} \mathbf{e}_{t, k}$ [29], where $\mathbf{e}_{t, k}$ is estimation error vector due to the time lag per transmit antenna, and its elements are complex random variables with zero mean and variance $\sigma_{e}$, and $\rho_{\tau_{t, k}}$ is the correlation coefficient $\left(\rho_{\tau_{t, k}} \in[0,1]\right.$, which it can be drawn from $\rho_{\tau_{t, k}}=\left[J_{0}\left(2 \pi f_{d_{t, k}} \tau_{d_{t, k}}\right)\right]^{2}$, where $J_{0}(x)$ denotes the zero order Bessel function of first kind [28], and $f_{d_{t, k}}$ denotes the Doppler frequency).

With arbitrary TAS, the PDF of $\gamma_{t, k}$ can be obtained as [30]

$$
\begin{aligned}
f_{\gamma_{t, k}}(x) & =u\left(\begin{array}{c}
N_{t} \\
u
\end{array}\right)\left[\mathcal{F}_{\gamma_{t, i, k}}(x)\right]^{u-1}\left[1-\mathcal{F}_{\gamma_{t, i, k}}(x)\right]^{N_{t}-u} \\
& \times f_{\gamma_{t, i, k}}(x) .
\end{aligned}
$$

The PDF of the delayed version $\hat{\gamma}_{t, k}$ of the true $\gamma_{t, k}$ is expressed as

$$
f_{\hat{\gamma}_{t, k}}(x)=\int_{0}^{\infty} f_{\hat{\gamma}_{t, k} \mid \gamma_{t, k}}(y \mid x) f_{\gamma_{t, k}}(x) d x
$$

where

$$
f_{\hat{\gamma}_{t, k} \mid \gamma_{t, k}}(y \mid x)=\frac{f_{\hat{\gamma}_{t, i, k}, \gamma_{t, i, k}}(y, x)}{f_{\gamma_{t, i, k}}(x)},
$$

and their joint PDF $f_{\hat{\gamma}_{t, i, k}, \gamma_{t, i, k}}(y, x)$ is given by [31]

$$
\begin{aligned}
f_{\hat{\gamma}_{t, i, k}, \gamma_{t, i, k}}(y, x) & =\left(\frac{1}{\bar{\gamma}_{D, t}^{\prime}}\right)^{L_{k}+1} \frac{e^{-\frac{x+y}{\left(1-\rho_{t, k}\right) \bar{\gamma}_{D, t}^{\prime}}}}{\left(1-\rho_{\tau_{t, k}}\right)} \\
& \times I_{L_{k}-1}\left(\frac{2 \sqrt{\rho_{\tau_{t, k}} x y}}{\bar{\gamma}_{D, t}^{\prime}\left(1-\rho_{\tau_{t, k}}\right)}\right) .
\end{aligned}
$$

The PDF and CDF of the delayed version $\hat{\gamma}_{t, k}$, which are referred to as $f_{\hat{\gamma}_{t, k}}(x)$ and $\mathcal{F}_{\hat{\gamma}_{t, k}}(x)$, respectively, can be obtained as shown in (35) and (36), respectively. Where $\zeta=\frac{\left(N_{t}-n\right)}{\bar{\gamma}_{D, t}^{\prime}\left[\left(N_{t}-n-1\right)\left(1-\rho_{\tau_{t, k}}\right)+1\right]}, c$ is a multi-index M-tuple having a length of $|c|=\sum_{p=0}^{L_{k}-1} c_{p}, v=\sum_{p=0}^{L_{k}-1} p c_{p}$, and $R(c, n)=\Gamma\left(N_{t}-n\right) \Pi_{p=0}^{L_{k}-1} \frac{1}{[\Gamma(p+1)] c_{p} \Gamma\left(c_{p}+1\right)}$.

\section{A. Exact Outage Probability}

1) Scenario A of Interference: The outage probability in this case can be expressed by conditioning the CDF of $\hat{\gamma}_{t, k}$ that is obtained in (36) on the interference distribution, and using the same steps adopted previously for SNR-based Post-TAS case. Consequently, using the series expansion of incomplete Gamma function $\gamma(n, x)=(n-1) !\left(1-e^{-x} \sum_{k=0}^{n-1} \frac{x^{k}}{k !}\right)$, and after some analytical manipulations, the resulting outage probability can be obtained as shown in (37), The term $\mathcal{J}_{4}$ in (37) is given in the following proposition.

Proposition 5: The term $\mathcal{J}_{4}$ is expressed as

$$
\mathcal{J}_{4}=(-1)^{j}\left(\zeta \gamma_{t h}\right)^{-j} H_{1,2}^{2,0}\left[\left.\alpha_{t}\left(\zeta \gamma_{t h}\right)^{\frac{2}{v}}\right|_{\left(j, \frac{2}{v}\right),(0,1)} ^{\left(0, \frac{2}{v}\right)}\right] \text {. }
$$

Proof: See Appendix E.

Substituting the resulting expression of $\operatorname{Pr}\left\{\hat{\gamma}_{\mathrm{SINR}, t, k}<\right.$ $\left.\gamma_{t h}\right\}$ for $t=\left\{\mathrm{M}_{B}, \mathrm{~F}_{B}\right\}$ and $k=\mathrm{M}_{U}$ in (16) yields the desired result for the outage probability of $\mathrm{M}_{U}$.

2) Scenario B of Interference: The outage probability has the form that is similar to that given in (37) but with $\mathcal{J}_{4}$ therein is replaced by $\mathcal{J}_{5}$, which is given the following proposition.

Proposition 6: The term $\mathcal{J}_{5}$ is expressed as

$$
\mathcal{J}_{5}=(-1)^{j}\left(\zeta \gamma_{t h}\right)^{-j} H_{1,2}^{2,0}\left[\left.\phi_{t}\left(\zeta \gamma_{t h}\right)^{\frac{2}{v}}\right|_{\left(j, \frac{2}{v}\right),(0,1)} ^{\left(0, \frac{2}{v}\right)}\right] \text {. }
$$

Proof: See Appendix E.

Inserting the resulting expression of $\operatorname{Pr}\left\{\tilde{\gamma}_{\mathrm{SINR}, t, k}<\gamma_{t h}\right\}$ for $t=\left\{\mathrm{M}_{B}, \mathrm{~F}_{B}\right\}$ and $k=\mathrm{M}_{U}$ in (18) yields the desired final result.

\section{B. Exact Average Error Rate}

1) Scenario A of Interference: The average error rate can be obtained using the MGF approach as shown in (21). In this case, after some analytical analysis, the resulting MGF of $\hat{\gamma}_{\text {SINR }, t, k}$, which is denoted as $\mathcal{M}_{\hat{\gamma}_{\mathrm{SINR}, t, k}}(s)$ can be obtained as shown in (40). Inserting the result in (40) into (21) yields the expression of $\mathrm{Pe}_{t, k}$, and now inserting it for $t=\left\{\mathrm{M}_{B}, \mathrm{~F}_{B}\right\}$ and $k=\mathrm{M}_{U}$ into (20) gives the desired result for the average error rate of $\mathrm{M}_{U}$.

2) Scenario $B$ of Interference: For this case, the term $\mathcal{M}_{\hat{\gamma}_{\text {SINR }, t, k}}(s)$ has the same expression as in (40) but after replacing $\alpha_{t}$ by $\phi_{t}$. Substituting the resulting expression of $\mathrm{Pe}_{t, k}$ for $t=\left\{\mathrm{M}_{B}, \mathrm{~F}_{B}\right\}$ and $k=\mathrm{M}_{U}$ into (24) yields the desired final result. 


$$
\begin{aligned}
f_{\hat{\gamma}_{t, k}}(x) & =u\left(\begin{array}{c}
N_{t} \\
u
\end{array}\right) \frac{1}{\Gamma\left(L_{k}\right)\left(1-\rho_{\tau_{t, k}}\right) \bar{\gamma}_{D, t}^{\prime}}\left(\frac{x}{\left(1-\rho_{\tau_{t, k}}\right) \bar{\gamma}_{D, t}^{\prime}}\right)^{L_{k}-1} \sum_{n=0}^{u-1}\left(\begin{array}{c}
u-1 \\
n
\end{array}\right)(-1)^{u-n-1} e^{-x \zeta} \sum_{|c|=N_{t}-n-1} \Gamma(v+1) \\
& \times R(c, n)\left(\frac{1-\rho_{\tau_{t, k}}}{\left(N_{t}-n-1\right)\left(1-\rho_{\tau_{t, k}}\right)+1}\right)^{v+L_{k}} \sum_{r=0}^{v}\left(\begin{array}{c}
v+L_{k}-1 \\
v-r
\end{array}\right) \frac{1}{\Gamma(r+1)}\left(\frac{\rho_{\tau_{t, k}}\left(1-\rho_{\tau_{t, k}}\right)^{-1} \zeta x}{N_{t}-n}\right)^{r},
\end{aligned}
$$

$$
\begin{aligned}
& \mathcal{F}_{\hat{\gamma}_{t, k}}(y)=u\left(\begin{array}{c}
N_{t} \\
u
\end{array}\right) \frac{1}{\Gamma\left(L_{k}\right)\left(1-\rho_{\tau_{t, k}}\right) \bar{\gamma}_{D, t}^{\prime}}\left(\frac{1}{\left(1-\rho_{\tau_{t, k}}\right) \bar{\gamma}_{D, t}^{\prime}}\right) \sum_{n=0}^{L_{k}-1}\left(\begin{array}{c}
u-1 \\
n
\end{array}\right)(-1)^{u-n-1} \sum_{|c|=N_{t}-n-1} \Gamma(v+1) R(c, n) \\
& \times\left(\frac{1-\rho_{\tau_{t, k}}}{\left(N_{t}-n-1\right)\left(1-\rho_{\tau_{t, k}}\right)+1}\right)^{v+L_{k}} \sum_{r=0}^{v}\left(\begin{array}{c}
v+L_{k}-1 \\
v-r
\end{array}\right) \frac{1}{\Gamma(r+1)}\left(\frac{\rho_{\tau_{t, k}}\left(1-\rho_{\tau_{t, k}}\right)^{-1} \zeta}{N_{t}-n}\right)^{r} \zeta^{-L_{k}-r} \gamma\left(L_{k}+r, \zeta y\right) .
\end{aligned}
$$

$$
\begin{aligned}
& \operatorname{Pr}\left\{\hat{\gamma}_{\mathrm{SINR}, t, k}<\gamma_{t h}\right\}=\left(\begin{array}{c}
N_{t} \\
u
\end{array}\right) \frac{u}{\Gamma\left(L_{k}\right)\left(1-\rho_{\tau_{t, k}}\right) \bar{\gamma}_{D, t}^{\prime}}\left(\frac{1}{\left(1-\rho_{\tau_{t, k}}\right) \bar{\gamma}_{D, t}^{\prime}}\right)^{L_{k}-1} \sum_{n=0}^{u-1}\left(\begin{array}{c}
u-1 \\
n
\end{array}\right)(-1)^{u-n-1} \\
& \times \sum_{|c|=N_{t}-n-1} \Gamma(v+1) R(c, n)\left(\frac{1-\rho_{\tau_{t, k}}}{\left(N_{t}-n-1\right)\left(1-\rho_{\tau_{t, k}}\right)+1}\right)^{v+L_{k}} \sum_{r=0}^{v}\left(\begin{array}{c}
v+L_{k}-1 \\
v-r
\end{array}\right)\left(\frac{\rho_{\tau_{t, k}}\left(1-\rho_{\tau_{t, k}}\right)^{-1}}{N_{t}-n}\right)^{r} \\
& \times \frac{1}{\Gamma(r+1)}\left(\frac{L_{k}+r-1 !}{\zeta^{L_{k}}}-e^{-\gamma_{t h} \zeta} \sum_{k=0}^{L_{k}+r-1} \frac{L_{k}+r-1 ! \zeta^{k-L_{k}} \gamma_{t h}^{k}}{k !} \sum_{j=0}^{k}\left(\begin{array}{c}
k \\
j
\end{array}\right) \mathcal{J}_{4}\right) .
\end{aligned}
$$

$$
\begin{aligned}
& \mathcal{M}_{\hat{\gamma}_{\mathrm{SIN}, t, k}}(s)=u\left(\begin{array}{c}
N_{t} \\
u
\end{array}\right) \frac{1}{\Gamma\left(L_{k}\right)\left(1-\rho_{\tau_{t, k}}\right) \bar{\gamma}_{D, t}^{\prime}}\left(\frac{1}{\left(1-\rho_{\tau_{t, k}}\right) \bar{\gamma}_{D, t}^{\prime}}\right)^{L_{k}-1} \sum_{n=0}^{u-1}\left(\begin{array}{c}
u-1 \\
n
\end{array}\right)(-1)^{u-n-1} \\
& \times \sum_{|c|=N_{t}-n-1} \Gamma(v+1) R(c, n)\left(\frac{1-\rho_{\tau_{t, k}}}{\left(N_{t}-n-1\right)\left(1-\rho_{\tau_{t, k}}\right)+1}\right)^{v+L_{k}} \sum_{r=0}^{v}\left(\begin{array}{c}
v+L_{k}-1 \\
v-r
\end{array}\right) \frac{1}{\Gamma(r+1)} \\
& \times\left(\frac{\rho_{\tau_{t, k}}\left(1-\rho_{\tau_{t, k}}\right)^{-1}}{N_{t}-n}\right)^{r}\left(\frac{L_{k}+r-1 !}{\zeta^{L_{k}}}-s \sum_{k=0}^{L_{k}+r-1} \frac{L_{k}+r-1 ! \zeta^{k-L_{k}}}{k !} \sum_{j=0}^{k}\left(\begin{array}{c}
k \\
j
\end{array}\right)\left(\frac{-1}{\zeta}\right)^{j}(s+\zeta)^{-k+j-1}\right. \\
& \left.\times H_{2,2}^{2,1}\left[\left.\alpha_{t} \zeta^{\frac{2}{v}}(s+\zeta)^{-\frac{2}{v}}\right|_{\left(j, \frac{2}{v}\right),(0,1)} ^{\left(j-k, \frac{2}{v}\right),\left(0, \frac{2}{v}\right)}\right]\right) .
\end{aligned}
$$

\section{Performance Analysis Using SINR-Based Post-TAS}

This Section extends the analysis of the outage probability and the average error rate of macrocell user of interest $\mathrm{M}_{U}$ by considering the SINR-Based Post-TAS. Moreover, it provides the compact asymptotic results for these performance measures in the high SNR regime.

\section{A. Exact Outage Probability}

1) Scenario A of Interference: Following the same steps in equations (16) and (54)-(55), the outage probability in this case can be expressed as in (41). where $(a)$ follows from the binomial formula $\left(1+\bar{\gamma}_{I, t}\right)^{l}=\sum_{j=0}^{l}\left(\begin{array}{l}l \\ j\end{array}\right) \bar{\gamma}_{I, t}^{j}$, and the term $\mathcal{J}_{6}$ is given the following proposition.

Proposition 7: The term $\mathcal{J}_{6}$ is expressed as

$$
\mathcal{J}_{6}=(-1)^{j}\left(\frac{\gamma_{t h}}{\bar{\gamma}_{D, t}^{\prime}}\right)^{-j} H_{1,2}^{2,0}\left[\left.\alpha_{t}\left(\frac{\gamma_{t h}}{\bar{\gamma}_{D, t}^{\prime}}\right)^{\frac{2}{v}}\right|_{\left(j, \frac{2}{v}\right),(0,1)} ^{\left(0, \frac{2}{v}\right)}\right] \text {. }
$$

Proof: See Appendix E.

Substituting the resulting expression of $\operatorname{Pr}\left\{\widetilde{\gamma}_{\text {SINR }, t, k}<\right.$ $\left.\gamma_{t h}\right\}$ for $t=\left\{\mathrm{M}_{B}, \mathrm{~F}_{B}\right\}$ and $k=\mathrm{M}_{U}$ in (16) yields the 


$$
\begin{aligned}
\operatorname{Pr}\left\{\widetilde{\gamma}_{\text {SINR }, t, k}<\gamma_{t h}\right\} & =\left[1-e^{\frac{-\gamma_{t h}}{\bar{\gamma}_{D, t}^{\prime}}} \sum_{l=0}^{L_{k}-1} \frac{\left(\frac{\gamma_{t h}}{\bar{\gamma}_{D, t}^{\prime}}\right)^{l}}{l !} \mathbb{E}_{\bar{\gamma}_{I, t}}\left\{\left(1+\bar{\gamma}_{I, t}\right)^{l} e^{-\frac{\bar{\gamma}_{I, t} \gamma_{t h}}{\bar{\gamma}_{D, t}}}\right\}\right]^{N_{t}}, \\
& \stackrel{(a)}{=}\left[1-e^{\frac{-\gamma_{t h}}{\bar{\gamma}_{D, t}^{\prime}}} \sum_{l=0}^{L_{k}-1} \frac{\left(\frac{\gamma_{t h}}{\bar{\gamma}_{D, t}^{\prime}}\right)^{l}}{l !} \sum_{j=0}^{l}\left(\begin{array}{l}
l \\
j
\end{array}\right) \mathbb{E}_{\bar{\gamma}_{I, t}}\left\{\bar{\gamma}_{I, t}^{j} e^{-\frac{\bar{\gamma}_{I, t} \bar{\gamma}_{t h}}{\overline{\bar{t}}_{D, t}^{\prime}}}\right\}\right]^{N_{t}}, \\
& =\left[1-e^{\frac{-\gamma_{t h}}{\bar{\gamma}_{D, t}^{\prime}}} \sum_{l=0}^{L_{k}-1} \frac{\left(\frac{\gamma_{t h}}{\bar{\gamma}_{D, t}^{\prime}}\right)^{l}}{l !} \sum_{j=0}^{l}\left(\begin{array}{l}
l \\
j
\end{array}\right) \mathcal{J}_{6}\right]^{N_{t}},
\end{aligned}
$$

desired result for the outage probability of $\mathrm{M}_{U}$.

2) Scenario $B$ of Interference: The outage probability in this case has a similar form to that given in (41) but with $\mathcal{J}_{6}$ therein is replaced by $\mathcal{J}_{7}$, which is given the following proposition.

Proposition 8: The term $\mathcal{J}_{7}$ is expressed as

$$
\mathcal{J}_{7}=(-1)^{j}\left(\frac{\gamma_{t h}}{\bar{\gamma}_{D, t}^{\prime}}\right)^{-j} H_{1,2}^{2,0}\left[\left.\phi_{t}\left(\frac{\gamma_{t h}}{{\overline{\gamma_{D, t}^{\prime}}}_{D,}^{\prime}}\right)^{\frac{2}{v}}\right|_{\left(j, \frac{2}{v}\right),(0,1)} ^{\left(0, \frac{2}{v}\right)}\right] \text {. }
$$

Proof: See Appendix E.

Substituting the resulting expression of $\operatorname{Pr}\left\{\widetilde{\gamma}_{\text {SINR }, t, k}<\right.$ $\left.\gamma_{t h}\right\}$ for $t=\left\{\mathrm{M}_{B}, \mathrm{~F}_{B}\right\}$ and $k=\mathrm{M}_{U}$ in (18) yields the desired final result.

\section{B. Exact Analysis of Error Rate}

1) Scenario A of Interference: The average error rate is obtained using the MGF method in (21), where the corresponding MGF of $\widetilde{\gamma}_{\mathrm{SINR}, t, k}$ in this case, which is denoted by $\mathcal{M}_{\widetilde{\gamma}_{\mathrm{SINR}, t, k}}(s)$ can be obtained as shown in (44), where $\Omega_{t}=\sum_{l=0}^{L_{k}-1} \frac{1}{l !} \sum_{j=0}^{l}\left(\begin{array}{l}l \\ j\end{array}\right)(-1)^{j}\left(\frac{1}{\bar{\gamma}_{D, t}^{\prime}}\right)^{l-j}$. Inserting the expression in (44) into (21) gives the expression of $\mathrm{Pe}_{t, k}$, and now inserting it in (20) for $t=\left\{\mathrm{M}_{B}, \mathrm{~F}_{B}\right\}$ and $k=\mathrm{M}_{U}$ gives the desired result for the average error rate of $\mathrm{M}_{U}$.

2) Scenario B of Interference: Herein, $\mathcal{M}_{\widetilde{\gamma}_{\mathrm{SINR}, t, k}}(s)$ has the same expression as in (44) but after replacing $\alpha_{t}$ by $\phi_{t}$. Then, substituting the resulting expression of $\mathrm{Pe}_{t, k}$ for $t=\left\{\mathrm{M}_{B}, \mathrm{~F}_{B}\right\}$ and $k=\mathrm{M}_{U}$ into (24) yields the desired final result.

\section{Asymptotic Results}

1) Asymptotic Outage Probability: The asymptotic outage probability with the use of SINR-Based Post-TAS is derived by employing the Taylor series expansion for exponentials and the asymptotic expansion of $\mathrm{H}$-function at $z=0$ [19, eq.1.8.1]. The corresponding results for different interference scenarios are treated separately in the following two parts. a) Scenario A of Interference: The asymptotic outage probability can be expressed as

$$
\mathrm{P}_{t, k}^{\infty} \approx\left(\frac{\gamma_{t h}}{\bar{\gamma}_{D, t}^{\prime}}\right)^{\Lambda_{t, k}} \frac{\alpha_{t}}{\left(L_{k} !\right)^{N_{t}}}
$$

where $\Lambda_{t, k}=\frac{2}{v} N_{t} L_{k}+N_{t}$. The achievable diversity and coding gain can now be obtained as: $G_{d}^{\text {Iden }}=\Lambda_{t, k}$, and $G_{c}^{\text {Iden }}=\left(\frac{\alpha_{t}}{{\overline{\gamma_{D, t}^{\prime}}}^{\prime}\left(L_{k} !\right)^{N_{t}}}\right)^{-\frac{1}{\Lambda_{t, k}}}$. Substituting the resulting expression of $\mathrm{P}_{t, k}^{\infty}$ for $t=\left\{\mathrm{M}_{B}, \mathrm{~F}_{B}\right\}$ and $k=\mathrm{M}_{U}$ in (16) yields the desired asymptotic result.

b) Scenario B of Interference: The asymptotic outage probability can be obtained by using the result in (41) after replacing $\mathcal{J}_{6}$ by $\mathcal{J}_{7}$. That gives

$$
\mathrm{P}_{t, k}^{\infty} \approx\left(\frac{\gamma_{t h}}{\bar{\gamma}_{D, t}^{\prime}}\right)^{\Lambda_{t, k}} \frac{\phi_{t}}{\left(L_{k} !\right)^{N_{t}}} .
$$

Substituting the resulting expression of $\mathrm{P}_{t, k}^{\infty}$ for $t=$ $\left\{\mathrm{M}_{B}, \mathrm{~F}_{B}\right\}$ and $k=\mathrm{M}_{U}$ in (18) yields the desired final result for asymptotic outage probability in this case. The achievable diversity and coding gain are: $G_{d}^{\text {Ind }}=\Lambda_{t, k}$, and $G_{c}^{\text {Ind }}=\left(\frac{\phi_{t}}{\bar{\gamma}_{D, t}^{\prime}\left(L_{k} !\right)^{N_{t}}}\right)^{-\frac{1}{\Lambda_{t, k}}}$. Similarly, the outage performance of this scenario is also dominated by the pass loss of the interferer. The systems under identical and independent interference scenarios have the same diversity gain, but they differ in terms of their associated SINR gap or coding gains. This SINR gap can be obtained as $\frac{G_{c}^{\text {Id }}}{G_{c}^{\text {Ind }}}=\left(\frac{\alpha_{t}}{\phi_{t}}\right)^{-\frac{1}{\Lambda_{t, k}}}$. This result indicates that, for the same outage probability level, the performance of identical interference scenario outperforms that of the independent interference scenario by $10\left(1 / \Lambda_{t, k}\right) \log \left(\alpha_{t} / \phi_{t}\right) \mathrm{dB}$.

2) Asymptotic Error Rate:

a) Scenario A of Interference: The asymptotic average error rate is obtained using the MGF approach in (21). 


$$
\mathcal{M}_{\widetilde{\gamma}_{\mathrm{SINR}, t, k}}(s)=s\left[\frac{1}{N_{t}}-\Omega_{t}\left(\frac{s}{N_{t}}+\frac{1}{\bar{\gamma}_{D, t}^{\prime}}\right)^{j-l-1} H_{2,2}^{2,1}\left[\left.\alpha_{t}\left(\frac{1}{\bar{\gamma}_{D, t}^{\prime}}\right)^{\frac{2}{v}}\left(\frac{s}{N_{t}}+\frac{1}{\bar{\gamma}_{D, t}^{\prime}}\right)^{-\frac{2}{v}}\right|_{\left(j, \frac{2}{v}\right),(0,1)} ^{\left(j-l, \frac{2}{v}\right),\left(0, \frac{2}{v}\right)}\right]\right]^{N_{t}},
$$

Herein, the asymptotic $\operatorname{MGF} \mathcal{M}_{t, k}^{\infty}(s)$ is written as

$$
\begin{aligned}
\mathcal{M}_{t, k}^{\infty}(s) & \approx \frac{\alpha_{t}}{\left(L_{k} !\right)^{N_{t}}} s^{-\left(\frac{2}{v} L_{k} N_{t}+N_{t}\right)} \Gamma\left(\frac{2}{v} L_{k} N_{t}+N_{t}+1\right) \\
& \times\left(\frac{1}{\bar{\gamma}_{D, t}^{\prime}}\right)^{\frac{2}{v} L_{k} N_{t}+N_{t}}
\end{aligned}
$$

Then, asymptotic average error rate can be obtained by inserting (47) into (21), which gives

$$
\begin{aligned}
\mathrm{Pe}_{t, k}^{\infty} & \approx \frac{\Gamma\left(\frac{2}{v} L_{k} N_{t}+N_{t}+1\right)}{\pi\left(L_{k} !\right)^{N_{t}}} \alpha_{t} \\
& \times\left(\frac{1}{\bar{\gamma}_{D, t}^{\prime} \sin ^{2}(\pi / 2)}\right)^{\frac{2}{v} L_{k} N_{t}+N_{t}} \mathcal{I}_{1} .
\end{aligned}
$$

where $\mathcal{I}_{1}$ is obtained as in (30). This yields the closedform expression of asymptotic average error rate. Inserting the resulting expression of $\mathrm{Pe}_{t, k}^{\infty}$ for $t=\left\{\mathrm{M}_{B}, \mathrm{~F}_{B}\right\}$ and $k=\mathrm{M}_{U}$ into (20) yields the desired final results.

b) Scenario $B$ of Interference: The expression for $\mathrm{Pe}_{t, k}^{\infty}$ is the same as in (48) after replacing $\alpha_{t}$ by $\phi_{t}$. Inserting the resulting expression of $\mathrm{Pe}_{t, k}^{\infty}$ for $t=\left\{\mathrm{M}_{B}, \mathrm{~F}_{B}\right\}$ and $k=\mathrm{M}_{U}$ into (24) yields the final results for the asymptotic average error rate of $\mathrm{M}_{U}$ in this case.

\section{NUMERICAL RESULTS AND PRACTICAL IMPLEMENTATION}

This Section presents some selected numerical results, which aim to support the analytical developments in the previous Sections and to explain the impact of various design and channel parameters on the achieved performance of the user of interest. Moreover, it overviews some related practical issues of the adopted MIMO systems herein in the context of latest wireless technologies.

\section{A. Numerical Results}

In this section, numerical results are provided to clarify some outcomes of the developed results in the previous sections. All results are presented assuming an SINR threshold of $\gamma_{t h}=5 \mathrm{~dB}$ except for Fig. 7. The obtained results are verified via Monte Carlo simulations, wherein the simulated points are obtained by averaging over $10^{5}$ independent trials.

Fig. 2 demonstrates the comparison between SNR-based selection and SINR-based selection outage probability results versus average SNR when the selection probability of $\operatorname{Pr}\left(\rho_{1}<1\right)=\operatorname{Pr}\left(\rho_{2}<1\right)=0.8$, the number

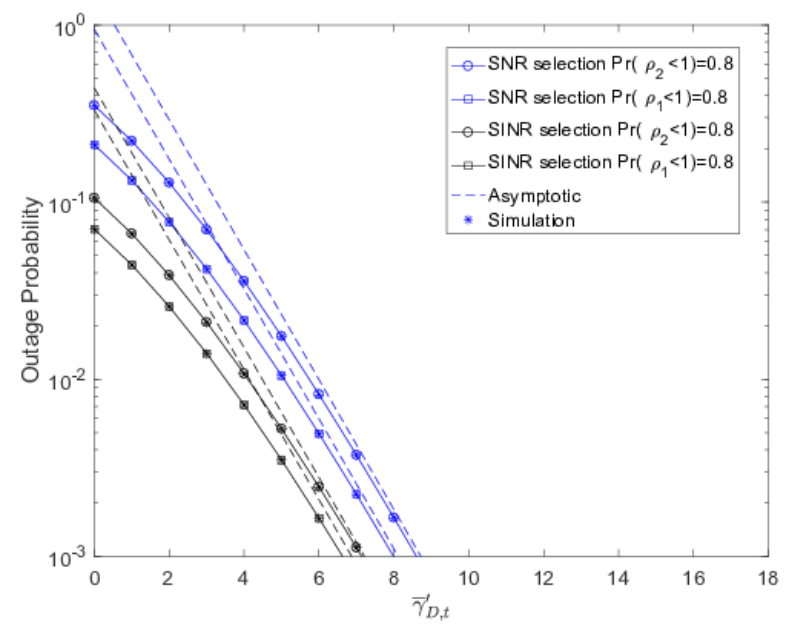

Fig. 2: Outage probability comparison between SNR-based and SINR-based TAS for TAS/MRC system under PPP interference and for different interference models on spatial links when $N_{t, k}=2$ and $L_{t, k}=2$.

of transmit antennas $N_{t, k}=2$, the number of receive antennas $L_{t, k}=2$, path loss exponent $v=3$, average INR of $20 \mathrm{db}$, and $\lambda_{t}=10^{-4}$. This reveals that when the association event $\operatorname{Pr}\left\{\rho_{k}>1\right\}$, where $k=1$ refers to identical interference and $k=2$ refers to un-correlated interference, $M_{U}$ chooses the femtocell link over the macrocell link. Otherwise it selects the marcocell link. It can be clearly seen that the SINR-based selection outperforms SNR-based selection. In addition, the performance for identical interference outperforms the independent interference scenario. Monte Carlo simulations are also provided, showing an excellent agreement with the developed analytical expressions and their associated asymptotic results.

Fig. 3 depicts the outage probability of SINR-based selection for identical interference scenario versus the average SNR. In particular, the outage probability is performed for different selection probability values $\operatorname{Pr}\left(\rho_{1}<\right.$ $1)=\{0.8,0.6,0.4\}$ and different values of $\alpha_{t}$. The figure shows that the outage probability is increased when the likelihood of the association event is decreased, which reveals that the macrocell user tends to use the macrocell resources over the femtocell when the interference is high on the femtocell link. In addition, it is obvious from the figure that increasing $\alpha_{t}$, which includes the interferers powers and path loss exponents, degrades the 


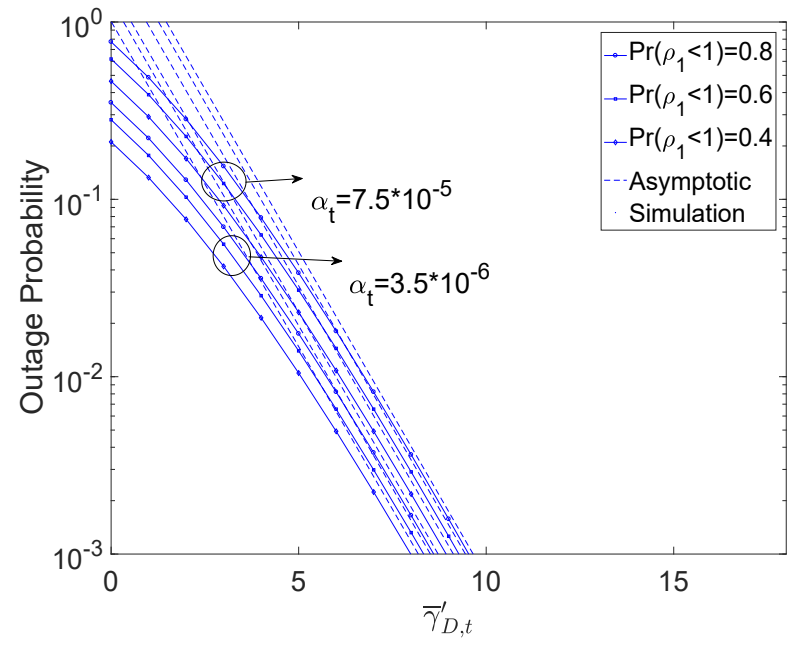

Fig. 3: The outage probability of SINR-based selection for different selection probability $\operatorname{Pr}\left(\rho_{1}<1\right)=\{0.8,0.6,0.4\}$ when $N_{t, k}=2$ and $L_{t, k}=2$.

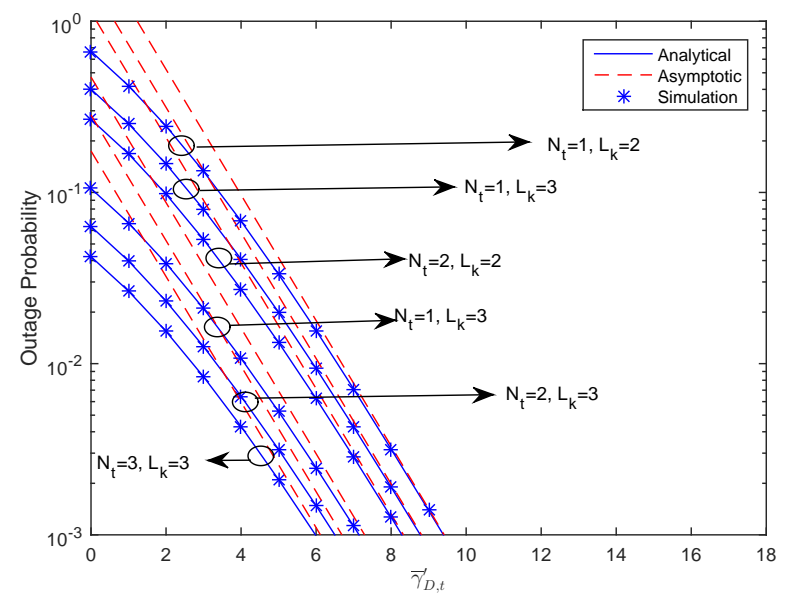

Fig. 4: The outage probability of SNR-based selection for both different $N_{t}=\{1,2,3\}$ transmit antennas and different receive antennas $L_{k}=\{2,3\}$ when $\operatorname{Pr}\left(\rho_{1}<1\right)=0.8$.

performance of the outage probability significantly.

Fig. 4 shows the outage probability of the SNR-based selection for identical interference case versus the average SNR. The results are performed for different number of transmit antennas $N_{t}=\{1,2,3\}$ and different number of receive antennas $L_{k}=\{2,3\}$, when selection probability $\operatorname{Pr}\left(\rho_{1}<1\right)=0.8$, path loss exponent $v=3$, average INR of $20 \mathrm{~dB}$, and $\lambda_{t}=10^{-4}$. This implies that increases more antennas at the transmitter and the receiver can enhance the performance of the outage probability.

Fig. 5 presents the average error rate performance of SNR-based selection for identical interference case versus the distance between macrocell and femtocell base stations and the macrocell user node $d_{t, k}$. The error

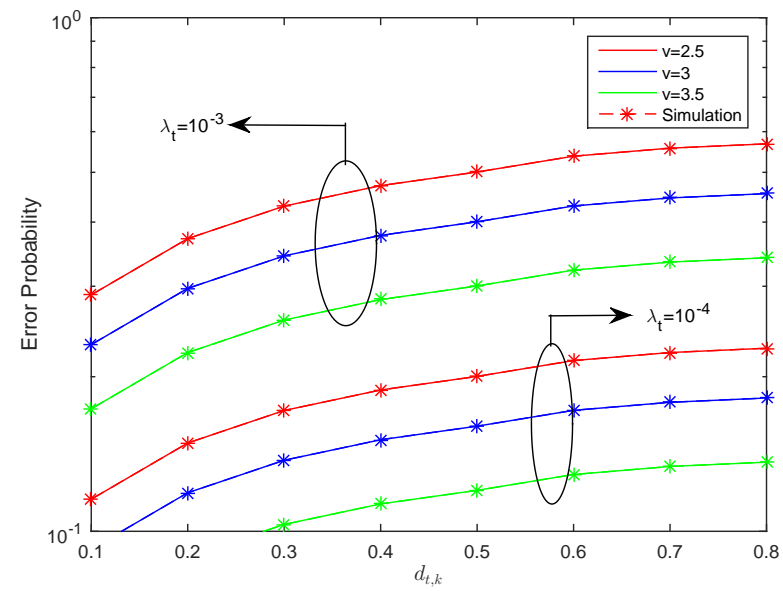

Fig. 5: The average error rate of SNR-based selection for different path loss exponent $v=\{2.5,3,3.5\}$ and intensity of interferers per unit area $\lambda_{t}=\left\{10^{-3}, 10^{-4}\right\}$ when $\operatorname{Pr}\left(\rho_{1}<\right.$ 1) $=0.8, N_{t}=2$, and $N_{r}=2$.

rate is plotted for different path loss exponent values $v=\{2.5,3,3.5\}$ when the selection probability $\operatorname{Pr}\left(\rho_{1}<\right.$ $1)=0.8$, the number of transmit antennas $N_{t}=2$, and number of receive antennas $L_{k}=2$, path loss exponent $v=3$, average INR of $20 \mathrm{~dB}$, and $\lambda_{t}=10^{-4}$ and modulation scheme of QPSK. A remarkable observation is that when the distance $d_{t, k}$ increases, the error rate rates increases, while the error rate deceases when the path loss exponent increases. Moreover, the error rate is significantly degraded with the increase of the density of interferer per unit area.

Fig. 6 depicts the delayed feedback outage probability performance for identical interference case versus the average SNR. The outage probability is performed for different $\rho_{\tau_{t, k}}=\{1,0.8,0.5,0.2\}, N_{t}=2, L_{k}=2$, path loss exponent $v=3$, average INR of $20 \mathrm{~dB}$, and $\lambda_{t}=10^{-4}$ and $\operatorname{Pr}\left(\rho_{1}<1\right)=0.8$, path loss exponent $v=3$, average INR of $20 \mathrm{~dB}$, and $\lambda_{t}=10^{-4}$. The outdated feedback have a significant impact on the overall performance, and hence it results in no diversity gain for both selection schemes. As can be readily observed, full diversity order is only achieved when $\rho_{\tau_{t, k}}=1$, which corresponds to the case with no feedback delay.

Fig. 7 shows the outage probability of the SNR-based selection for identical interference case versus the average SNR. The results are performed for different number of transmit antennas $N_{t}=\{2,3\}$ and different $\gamma_{t h}=$ $\{0,5\} \mathrm{dB}$, when selection probability $\operatorname{Pr}\left(\rho_{1}<1\right)=0.8$, path loss exponent $v=3, L k=\{3\}$, average INR of $20 \mathrm{~dB}$, and $\lambda_{t}=10^{-4}$. This implies the same results observed in Fig. 4 having different number of antennas at the transmitter. Furthermore, it is also observed that 


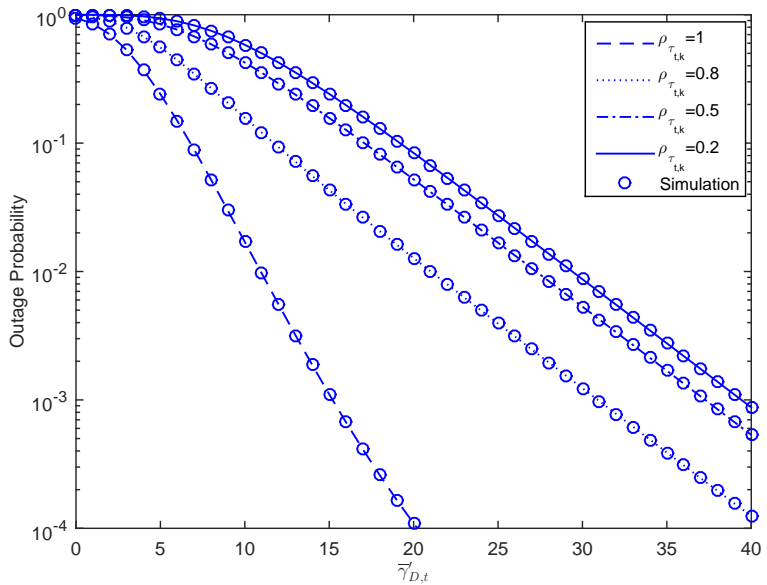

Fig. 6: The impact of feedback delay on the outage probability of SNR-based selection scheme for different correlation factor $\rho_{\tau_{t, k}}=\{1,0.8,0.5,0.2\}$ when $N_{t}=2, L=2$, and $\operatorname{Pr}\left(\rho_{1}<\right.$ 1) $=0.8$.

increasing the $\gamma_{t} h$ degrade the performance by increasing the outage probability.

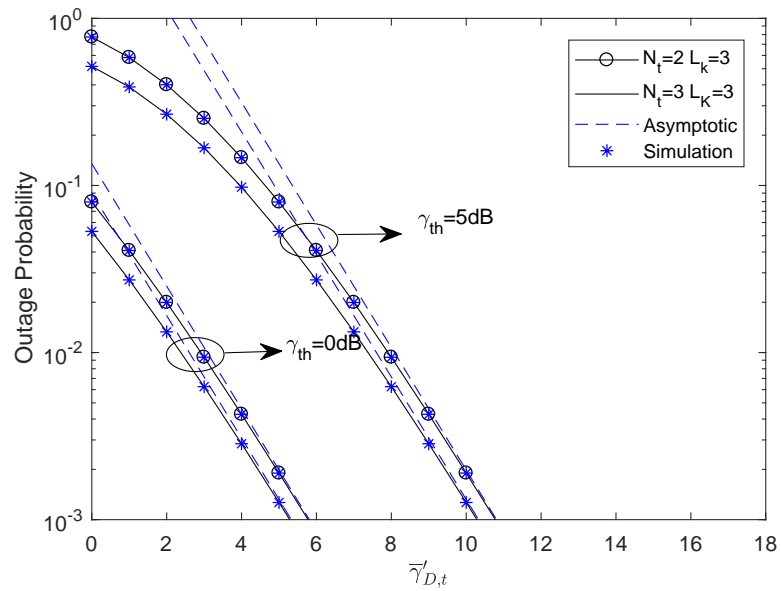

Fig. 7: The outage probability of SNR-based selection for both different $N_{t}=\{2,3\}$ transmit antennas and different threshold $\gamma_{t h}=\{0,5\}$

\section{B. Practical Implementation Issues}

The advantage of achieving full diversity at both transceiver sides by employing variants of MIMO systems provides motivation for TAS usage in LTE/LTE-A systems. In LTE system, the base station (or eNodeB) uses two antennas for transmission and reception, while the user equipment (or UE) uses two antennas in receive mode and only one antenna in transmit mode to avoid expanded energy drain in UE. Therefore, using TAS in the uplink is an attractive technology since it combines the gain of having one transmit antenna used at a time in the UE while benefit from the spatial diversity offered by the two antennas physically exist in UE. The OFDM signal in LTE requires reference signals (or called pilots) and they are used for channel estimation. In LTE uplink transmission, two types of pilot transmissions are used, sounding RS (SRS) and demodulation RS (DMRS). TAS occurs over two phases in LTE. In the first phase, the UE alternates transmission of SRS or DMRS from its two antennas. The eNodeB then estimates channel response of the UE from all its antennas and chooses the best. In the second phase, the eNodeB sends one bit feedback to the UE indicating the selected antenna [32].

There are few challenges using TAS, which are mainly in the phase of channel estimation using SRS and DMRS. The ability of tracking the variations in channel response decreases due to the fact that fewer SRS and DMRS are sent when using TAS which may results in an accurate channel estimation. More details can be found in [32].

\section{COnclusions}

This paper has presented new formulated problems that integrate different processing designs and network conditions in two-tier overlaid cellular networks. It analyzed the downlink performance of proposed hybrid controlaccess schemes for spectrum-shared two-tier MIMO networks with the use of TAS/MRC in the presence of PPP interference fields on spatial links. The treatment has covered detailed descriptions of the mode of operations of SINR-based Post-TAS and Pre-TAS interferenceaware hybrid control-access schemes. Thereafter, new analytical results for some important performance measures of a desired user have been developed, including the outage probability and average error rate under PPP interference fields on spatial links. These results have been presented for two TAS approaches, which are SNR-based and SINR-based selection. The expected performance enhancement of SINR-based selection with respect to the SNR-based selection have been discussed and quantified. In addition, asymptotic approximations for the considered performance measures have been obtained, which have been used to characterize key performance indicators, such as the diversity gain and coding gain for the two TAS approaches. The treatment has been extended to cover the practical issue of delayed TAS due to imperfect feedback channel and arbitrary TAS scenario, and it has investigated the practical cases of identical and un-correlated aggregated interference on transmit channels. The developed models and their associated analysis herein have investigated an integrated problem that deals with practical issues of resources 
control-access strategy, interference modeling and characterization, MIMO links processing, transmit selection approaches, and delayed feedback effect. They provide new enhancements on previous related works in terms of practical applicability and scope of applications.

\section{APPENDIX A}

Calculation of $\operatorname{Pr}\left\{\bar{\rho}_{\text {SINR }}<1\right\}$ AND $\operatorname{Pr}\left\{\rho_{1}<1\right\}$

This Appendix explains the evaluation of the results in (10) and (14). Specifically, the term $\operatorname{Pr}\left\{\rho_{1}<1\right\}$ is evaluated as $\operatorname{Pr}\left\{\rho_{1}<1\right\}=\operatorname{Pr}\left\{\eta I_{\mathrm{M}_{B}}-I_{\mathrm{F}_{B}}<0\right\}$, where $\eta=\eta_{0} N_{\mathrm{M}_{B}} / N_{\mathrm{F}_{B}}$. To proceed with the analysis, the Laplace transform of $I_{\mathrm{M}_{B}}$ (or $I_{\mathrm{F}_{B}}$ ) that follows the form in (3), can be re-written as [18]

$$
\begin{aligned}
\psi_{I_{t}}(s) & =\exp \left[-\lambda_{t}\left(s \bar{\gamma}_{I, t}\right)^{\frac{2}{v}} \beta(v)\right], \\
& \stackrel{(a)}{=} H_{0,1}^{1,0}\left[\left.\lambda_{t}\left(s \bar{\gamma}_{I, t}\right)^{\frac{2}{v}} \beta(v)\right|_{(0,1)} ^{-}\right],
\end{aligned}
$$

where $\bar{\gamma}_{I, t}=\bar{\gamma}_{I, t, i, \mathrm{M}_{U}}$ in this case, and (a) follows from the definition of Fox H-function [19]. The PDF of $I_{t}$ can be obtained as the inverse Laplace transform of (49) to give

$$
\begin{aligned}
f_{I_{t}}(y) & =\frac{1}{j 2 \pi} \lim _{T \rightarrow \infty} \int_{\epsilon-j T}^{\epsilon+j T} e^{s y} \psi_{I_{t}}(s) d s \\
& =\frac{1}{y} H_{1,1}^{1,0}\left[\left.\lambda_{t}\left(y \bar{\gamma}_{I, t}\right)^{\frac{2}{v}} \beta(v)\right|_{(0,1)} ^{\left(0, \frac{v}{2}\right)}\right] .
\end{aligned}
$$

Moreover, the CDF of $I_{t}$ can now be obtained as [23] $F_{I_{t}}(x)=\int_{0}^{x} f_{I_{t}}(y) d y=H_{1,1}^{1,0}\left[\left.\lambda_{t}\left(x \bar{\gamma}_{I, t}\right)^{\frac{2}{v}} \beta(v)\right|_{(0,1)} ^{\left(1, \frac{v}{2}\right)}\right]$.

Using the preceding results, $\operatorname{Pr}\{\rho<1\}$ can now be expressed using [24, eq. 4.12] as in (14).

\section{APPENDIX B}

\section{Calculation of $\operatorname{Pr}\left\{\rho_{2}<1\right\}$}

The term $\operatorname{Pr}\left\{\rho_{2}<1\right\}$ is evaluated as

$$
\begin{aligned}
\operatorname{Pr}\left\{\rho_{2}<1\right\}= & \int_{0}^{\infty} F_{\sum_{i=1}^{N_{\mathrm{M}_{B}}} I_{\mathrm{M}_{B}, i, M_{U}}}\left(\frac{x}{\eta_{0}}\right) \\
& \times f_{\sum_{i=1}^{N_{\mathrm{F}_{B}}} I_{\mathrm{F}_{B}, i, M_{U}}}(x) d x .
\end{aligned}
$$

where $\psi_{\sum_{i=1}^{N_{t}} I_{t, i}}(s)=\prod_{i=1}^{N_{t}} \exp \left[-\lambda_{t}\left(s \bar{\gamma}_{I, t, i}\right)^{\frac{2}{v}} \beta(v)\right]=$ $H_{0,1}^{1,0}\left[\left.\lambda_{t} s^{\frac{2}{v}}\left(\sum_{i=1}^{N_{t}}\left(\bar{\gamma}_{I, t, i}\right)^{\frac{2}{v}}\right) \beta(v)\right|_{(0,1)} ^{-}\right]$. Then it can be shown that the PDF and CDF of $\sum_{i=1}^{N_{t}} I_{t, i, M_{U}}$ have similar forms to those given in (50) and (51), respectively, but with the parameter $\left(\bar{\gamma}_{I, t}\right)^{\frac{2}{v}}$ therein is replaced by $\sum_{i=1}^{N_{t}}\left(\bar{\gamma}_{I, t, i}\right)^{\frac{2}{v}}$. Therefore, the result for $\operatorname{Pr}\left\{\rho_{2}<1\right\}$ can be expressed as in (15).

\section{APPENDIX C}

Calculation of $\operatorname{Pr}\left\{\gamma_{\text {SINR }, t, k}<\gamma_{t h}\right\}$ FOR SCENARIO A OF INTERFERENCE

The term $\operatorname{Pr}\left\{\gamma_{\mathrm{SINR}, t, k}<\gamma_{t h}\right\}$ can be expressed as

$$
\operatorname{Pr}\left\{\gamma_{\mathrm{SINR}, t, k}<\gamma_{t h}\right\}=\int_{0}^{\infty} F_{X}\left((1+y) \gamma_{t h}\right) f_{I_{t, k}}(y) d y,
$$

where $X=\max _{i=1, \ldots, N_{t}}\left\{\gamma_{\mathrm{SNR}, t, i, k}\right\}$ and $\gamma_{\mathrm{SNR}, t, i, k}=$ $\bar{\gamma}_{D, t}^{\prime} \sum_{r=1}^{L_{k}} \alpha_{t, i, k, r}^{2}$ as defined after (4). Then $\gamma_{\mathrm{SNR}, t, i, k}$ has a Gamma distribution with parameters $\gamma_{\mathrm{SNR}, t, i, k} \sim$ $\mathcal{G}\left(L_{k}, 1 / \bar{\gamma}_{D, t}^{\prime}\right)$. Therefore the CDF of $X$ can be expressed as

$$
F_{X}(x)=\prod_{i=1}^{N_{t}} \frac{1}{\Gamma\left(L_{k}\right)} \gamma\left(L_{k}, \frac{x}{\bar{\gamma}_{D, t}^{\prime}}\right),
$$

where $\gamma(a, x)$ is the lower incomplete Gamma function. Since $L_{k}$ is an integer, and using the finite sum representation of $\gamma(a, x)$, the result in (54) can be expanded as in (55) where $\stackrel{(a)}{=}$ follows from binomial expansion and $r_{\text {sum }}=\sum_{p=1}^{n}\left(r_{p}-1\right)$. Using the resulting expression in (55) into (53) gives the result in (17), where the term $\mathcal{J}_{1}$ is defined as

$$
\begin{aligned}
\mathcal{J}_{1} & =\int_{0}^{\infty} y^{j} e^{-\left(n \gamma_{t h} / \bar{\gamma}_{D, t}^{\prime}\right) y} f_{I_{t, k}}(y) d y \\
& \left.\stackrel{(a)}{=}(-1)^{j}\left[\frac{d^{j} \psi_{I_{t, k}}(s)}{d s^{j}}\right]\right|_{s=\left(\frac{n \gamma_{t h}}{\bar{\gamma}_{D, t}}\right)} \\
& \stackrel{(b)}{=}(-1)^{j}\left(\frac{n \gamma_{t h}}{\bar{\gamma}_{D, t}^{\prime}}\right)^{-j} H_{1,2}^{2,0}\left[\left.\alpha_{t}\left(\frac{n \gamma_{t h}}{\bar{\gamma}^{\prime D, t}}\right)^{\frac{2}{v}}\right|_{\left(j, \frac{2}{v}\right),(0,1)} ^{\left(0, \frac{2}{v}\right)}\right],
\end{aligned}
$$

where $\alpha_{t}=\lambda_{t} \beta(v) \bar{\gamma}_{I, t}^{\frac{2}{v}}$, (a) follows from the Laplace transform property [25, eq.(1.1.2.9)], and (b) follows from the derivative property of Fox H-function [19, eq.2.2.2].

\section{APPENDIX D}

Calculation OF $\operatorname{Pr}\left\{\gamma_{\text {SINR }, t, k}<\gamma_{t h}\right\}$ FOR SCENARIO

\section{B OF INTERFERENCE}

The term $\operatorname{Pr}\left\{\gamma_{\mathrm{SINR}, t, k}<\gamma_{t h}\right\}$ in (18) for $t=\left\{\mathrm{M}_{B}, \mathrm{~F}_{B}\right\}$ and $k=\mathrm{M}_{U}$ can be expressed as

$$
\begin{aligned}
\operatorname{Pr}\left\{\gamma_{\text {SINR }, t, k}<\gamma_{t h}\right\} & =\sum_{i=1}^{N_{t}} p_{i} \int_{0}^{\infty} F_{X}\left((1+y) \gamma_{t h}\right) \\
& \times f_{I_{t, i, k}}(y) d y
\end{aligned}
$$

where $f_{I_{t, i, k}}(x)$ is the PDF of $I_{t, i, k}$, evaluated as $f_{I_{t, i, k}}(x)=\frac{1}{\Gamma\left(L_{k}\right)}\left(\frac{1}{\bar{\gamma}_{D, t}^{\prime}}\right)^{L_{k}} x^{L_{k}-1} e^{-x / \bar{\gamma}_{D, t}^{\prime}}$, and the term $X$ has the same CDF that is given in (55). Moreover, the term $p_{i}$ denotes the probability that the $i$ th 


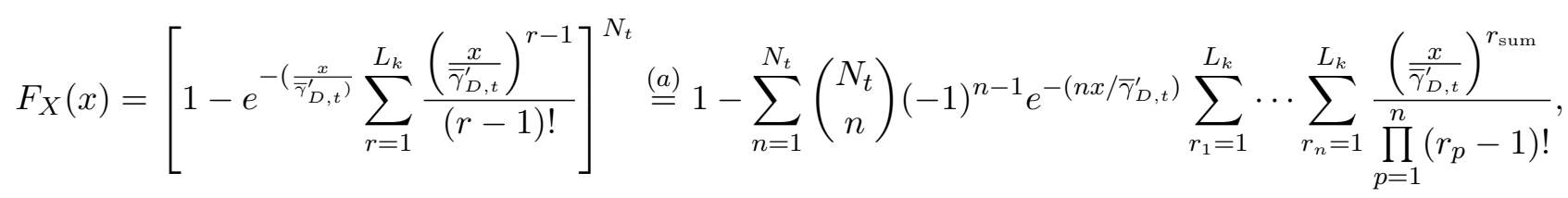

transmit antenna is selected and hence the associated interference power $I_{t, i, k}$ is observed. This term can be obtained as the probability of the following event: $\gamma_{\mathrm{SNR}, t, i, k}>\gamma_{\mathrm{SNR}, t, q, k}$, for $q=1,2, \ldots, N_{t}$ and $q \neq i$. Then $p_{i}$ is given as in (58). Expanding the brackets gives the expression in (59). Where $\mathcal{J}_{2}=\frac{\left(\frac{1}{\bar{\gamma}_{D, t}}\right)^{r_{\mathrm{sum}}+L_{k}}}{\Gamma\left(L_{k}\right)} \int_{0}^{\infty} e^{\left.\frac{-(x(n+1)}{\bar{\gamma}_{D, t}^{\prime}}\right)} x^{r_{\mathrm{sum}}+L_{k}-1} d x=$ $\frac{\Gamma\left(r_{\text {sum }}+L_{k}\right)}{\Gamma\left(L_{k}\right)}\left(\frac{1}{n+1}\right)^{r_{\text {sum }}+L_{k}}$. The evaluation of (57) follows the same procedure adopted to compute (53). The final result has a form that is similar to that given in (17) but with $\mathcal{J}_{1}$ therein is replaced by

$$
\begin{aligned}
\mathcal{J}_{3} & =\int_{0}^{\infty} y^{j} e^{-\left(n \gamma_{t h} / \bar{\gamma}_{D, t}^{\prime}\right) y} f_{I_{t, i, k}}(y) d y, \\
& \left.\stackrel{(a)}{=}(-1)^{j}\left[\frac{d^{j} \psi_{I_{t, k}}(s)}{d s^{j}}\right]\right|_{s=\left(n \gamma_{t h} / \bar{\gamma}_{D, t}^{\prime}\right)} \\
& \stackrel{(b)}{=}(-1)^{j}\left(\frac{n \gamma_{t h}}{\bar{\gamma}_{D, t}^{\prime}}\right)^{-j} H_{1,2}^{2,0}\left[\left.\phi_{t}\left(\frac{n \gamma_{t h}}{\bar{\gamma}_{D, t}^{\prime}}\right)^{\frac{2}{v}}\right|_{\left(j, \frac{2}{v}\right),(0,1)} ^{\left(0, \frac{2}{v}\right)}\right],
\end{aligned}
$$

where $\phi_{t}=\lambda_{t} \beta(v) \sum_{i=1}^{N_{t}}\left(\bar{\gamma}_{I, t, i}\right)^{\frac{2}{v}},(a)$ follows from the Laplace transform property [25, eq.(1.1.2.9)], and $(b)$ follows from the derivative property of Fox H-function [19, eq.2.2.2].

\section{APPENDIX E}

Calculation of $\mathcal{J}_{4}, \mathcal{J}_{5} \mathcal{J}_{6}$, AND $\mathcal{J}_{7}$

The term $\mathcal{J}_{4}$ can be evaluated as in (61).

$$
\begin{aligned}
\mathcal{J}_{4} & =\int_{0}^{\infty} y^{j} e^{-\zeta \gamma_{t h} y} f_{I_{t, k}}(y) d y \\
& =\left.(-1)^{j}\left[\frac{d^{j} \psi_{I_{t, k}}(s)}{d s^{j}}\right]\right|_{s=\zeta \gamma_{t h}}, \\
& =(-1)^{j}\left(\zeta \gamma_{t h}\right)^{-j} H_{1,2}^{2,0}\left[\left.\alpha_{t}\left(\zeta \gamma_{t h}\right)^{\frac{2}{v}}\right|_{\left(j, \frac{2}{v}\right),(0,1)} ^{\left(0, \frac{2}{v}\right)}\right] .
\end{aligned}
$$

The term $\mathcal{J}_{5}$ can be evaluated as

$$
\begin{aligned}
\mathcal{J}_{5} & =\int_{0}^{\infty} y^{j} e^{-\zeta \gamma_{t h} y} f_{I_{t, k}}(y) d y \\
& =\left.(-1)^{j}\left[\frac{d^{j} \psi_{I_{t, k}}(s)}{d s^{j}}\right]\right|_{s=\zeta \gamma_{t h}}, \\
& =(-1)^{j}\left(\zeta \gamma_{t h}\right)^{-j} H_{1,2}^{2,0}\left[\left.\phi_{t}\left(\zeta \gamma_{t h}\right)^{\frac{2}{v}}\right|_{\left(j, \frac{2}{v}\right),(0,1)} ^{\left(0, \frac{2}{v}\right)}\right] .
\end{aligned}
$$

The term $\mathcal{J}_{6}$ can be evaluated as

$$
\begin{aligned}
\mathcal{J}_{6} & =\int_{0}^{\infty} y^{j} e^{-\left(\gamma_{t h} / \bar{\gamma}_{D, t}^{\prime}\right) y} f_{I_{t, k}}(y) d y, \\
& =\left.(-1)^{j}\left[\frac{d^{j} \psi_{I_{t, k}}(s)}{d s^{j}}\right]\right|_{s=\left(\frac{\gamma_{t h}}{\bar{\gamma}_{D, t}^{\prime}}\right)}, \\
& =\left(\frac{\gamma_{t h}}{-\bar{\gamma}_{D, t}^{\prime}}\right)^{-j} H_{1,2}^{2,0}\left[\left.\alpha_{t}\left(\frac{\gamma_{t h}}{\bar{\gamma}_{D, t}^{\prime}}\right)^{\frac{2}{v}}\right|_{\left(j, \frac{2}{v}\right),(0,1)} ^{\left(0, \frac{2}{v}\right)}\right] .
\end{aligned}
$$

The term $\mathcal{J}_{7}$ can be evaluated as

$$
\begin{aligned}
\mathcal{J}_{7} & =\int_{0}^{\infty} y^{j} e^{-\left(\gamma_{t h} / \bar{\gamma}_{D, t}^{\prime}\right) y} f_{I_{t, i, k}}(y) d y, \\
& =\left.(-1)^{j}\left[\frac{d^{j} \psi_{I_{t, k}}(s)}{d s^{j}}\right]\right|_{s=\left(\frac{\gamma_{t h}}{\bar{\gamma}_{D, t}^{\prime}}\right)}, \\
& =\left(\frac{\gamma_{t h}}{-\bar{\gamma}_{D, t}^{\prime}}\right)^{-j} H_{1,2}^{2,0}\left[\left.\phi_{t}\left(\frac{\gamma_{t h}}{\bar{\gamma}_{D, t}^{\prime}}\right)^{\frac{2}{v}}\right|_{\left(j, \frac{2}{v}\right),(0,1)} ^{\left(0, \frac{2}{v}\right)}\right] .
\end{aligned}
$$

\section{REFERENCES}

[1] L. Dai, S. Zhou, Y. Yao, "Capacity analysis in CDMA distributed antenna systems“ IEEE Trans. Wireless Commun., pp. 2613-2620, vol. 4, no. 6, Nov. 2005.

[2] J. G. Andrews, S. Buzzi, W. Choi, S. V. Hanly, A. Lozano, A. C. K. Soong, and J. C. Zhang,"What will 5G be?" IEEE Jour. select. Areas Commun., pp. 1065-1082, vol. 32, no. 6, Jun. 2014.

[3] D. In Kim, E.-H. Shin, and M. S. Jin, "Hierarchical power control with interference allowance for uplink transmission in twotier heterogeneous networks," IEEE Trans. Wirless Commun., vol. 14, no. 2, Feb. 2015.

[4] Y. Ma, T. Lv, and Y. Lu, "Efficient power control in heterogeneous femto-macro cell networks," In Proc. IEEE WCNC, pp. 4215-4219, Apr. 2013.

[5] W. Pramudito, and Emad Alsusa, "A hybrid resource management technique for energy and QoS optimization in fractional frequency reuse based cellular networks, “ IEEE Trans Commun., vol. 61, no. 12, Dec. 2013.

[6] P. A. Dmochowski, P. J. Smith, and M. Shafi, "Capacity analysis for closed and open access femtocell networks," In Proc. IEEE PIMRC, pp. 1722-1727, Sept. 2012. 


$$
p_{i}=\int_{0}^{\infty}\left[\prod_{q=1, q \neq i}^{N_{t}} \operatorname{Pr}\left\{\gamma_{\mathrm{SNR}, t, q, k}<x\right\}\right] f_{\gamma_{\mathrm{SNR}, t, i, k}}(x) d x=\int_{0}^{\infty}\left[1-e^{-\left(\frac{x}{\left.\bar{\gamma}_{D, t}^{\prime}\right)}\right.} \sum_{r=1}^{L_{k}} \frac{\left(\frac{x}{\bar{\gamma}_{D, t}^{\prime}}\right)^{r-1}}{(r-1) !}\right]^{N_{t}-1} f_{\gamma_{\mathrm{SNR}, t, i, k}}(x) d x
$$

$$
p_{i}=1-\sum_{n=1}^{N_{t}-1}\left(\begin{array}{c}
N_{t} \\
n
\end{array}\right)(-1)^{n-1} \sum_{r_{1}=1}^{L_{k}} \cdots \sum_{r_{n}=1}^{L_{k}} \frac{\mathcal{J}_{2}}{\prod_{p=1}^{n}\left(r_{p}-1\right) !}
$$

[7] H. Bolcskei, R. U. Nabar, O. Oyman, and A. J. Paulraj, "Capacity scaling laws in MIMO relay networks," IEEE Trans. Wireless Commun., vol. 5, pp. 1433-1444, Jan. 2007.

[8] A. Forenza, M. R. McKay, A. Pand haripande, R. W. Heath, and I. B. Collings, "Adaptive MIMO transmission for Exploiting the Capacity of Spatially Correlated Channels," IEEE Trans. Veh. Technol., vol. 56, no. 2, pp. 619-630, Mar. 2007.

[9] A. F. Molisch and M. Z. Win, "MIMO systems with antenna selection," IEEE Microwave, vol. 5, no. 1, pp. 46-56, Mar. 2004.

[10] Z. Chen, "Asymptotic performance of transmit antenna selection with maximal-ratio combining for generalized selection criterion,“ IEEE Commun. Lett., vol. 8, no. 4, pp. 247-249, Apr. 2004.

[11] R. M. Radaydeh, "Performance analysis of rectangular quadrature amplitude modulation with combined arbitrary transmit antenna selection and receive maximal ratio combining in nakagami-m fading," IET Commun., vol. 2, no. 8, pp. 10771088, Sept. 2008.

[12] P. L. Yeoh, M. Elkashlan, and I. B. Collings, "Exact and asymptotic SER of distributed TAS/MRC in MIMO relay networks," IEEE Trans. Wireless Commun., vol. 10, no. 3, pp. 751-756, Mar. 2011.

[13] R. M. Radaydeh, "Performance of cellular mobile systems employing SNR-based GSC in presence of Rayleigh and Nakagami-q cochannel interferers," IEEE Trans. Veh. Technol., vol. 58, no. 6, pp. 3081-3088, Jul. 2009.

[14] R. M. Radaydeh, "SNR and SINR-based selection combining algorithms in the presence of arbitrarily distributed co-channel interferers," IET Commun., vol. 3, no. 1, pp. 57-66, Jan. 2009.

[15] E. S. Sousa, "Performance of a spread spectrum packet radio network link in a Poisson field of interferers, "IEEE Trans. Inf. Theory, vol. 38, pp. 1743-1754, Nov. 1992.

[16] T. X. Brown, "Cellular performance bounds via shotgun cellular systems,“'IEEE Jour. Select. Areas Commun., vol. 18, no. 11, pp. 2334-2455, Nov. 2000.

[17] D. Chen, T. Jiang, and Z. Zhang, "Frequency partitioning pethods to mitigate cross-tier interference in two-tier femtocell networks,“ IEEE Trans. Veh. Technol., vol. 64, no. 5, pp. 17931805, May 2015.

[18] M. Z. Win, P. C. Pinto, and L. A. Shepp, "A mathematical theory of network interference and its applications, “ Proc. IEEE, vol. 97, no. 2, pp. 205-230, Feb. 2009.

[19] A. M. Mathai, R. K. Saxena, and H. J. Haubold, The $H$ Function: Theory and Applications, 1st ed., London, New York: Springer Science, 2009.

[20] M. D. Renzo, C. Merola, A. Guidotti, F. Santucci, and G. E. Corazza, "Error performance of multi-antenna receivers in a poisson field of interferers: a stochastic geometry approach," IEEE Trans. Commu., vol. 61, no. 5, May 2013.
[21] Y. Chen and C. Tellambura,"Distribution functions of selection combiner output in equally correlated Rayleigh, Rician, and Nakagami-m fading channels," IEEE Trans. Commun., pp. 1948-1956, no. 11, vol. 52, 2004.

[22] A. M. Magableh, R. M. Radaydeh, and M.-S. Alouini, "On the performance of shared access control strategy for femtocells," Trans. Emerging Telecom. Technol., vol. 24, no. 2, 2013.

[23] V. A. Aalo, K. P. Peppas, G. Efthymoglou, M. Alwakeel, and S. Alwakeel, "Serial amplify-and-forward relay transmission systems in Nakagami-m fading channels with a poisson interference field,“ IEEE Trans. Veh. Technol., vol. 63, no. 5, Jun. 2014.

[24] D. willinger and S. Kokoska, CRC Standard Probability and Statistics Tables and Formulae, CRC, 2000.

[25] A. P. Prudnikov, Y. A. Brychkov, and O. I. Marichev, Integrals and Series Vol. 4: Direct Lapalce Transforms, 1992.

[26] M. K. Simon and M.-S. Alouini, Digital communication over fading channels, 2nd ed., Wiley, 2005.

[27] M. R. McKay, A. Zanella, I. B. Collings, and M. Chiani, "Error probability and SINR analysis of optimum combining in Rician fading," IEEE Trans. Commun., vol. 57, no. 3, pp. 676-687, Mar. 2009.

[28] I. S. Gradshteyn and I. M. Ryzhik, Table of Integrals, Series and Products, 7th ed., New York: Academic, 2007.

[29] R. M. Radaydeh, "MRC in Presence of asynchronous coChannel interference over frequency-selective Rayleigh fading channels," IEEE Trans. Veh. Technol., vol. 58, no. 8, pp. 4329 4341, Oct. 2009.

[30] R. M. Radaydeh, "Impact of delayed arbitrary transmit antenna selection on the performance of rectangular QAM with receive MRC in fading channel,“ IEEE Commun. Lett., vol.13, no.6, pp.390-392, 2009.

[31] R. M. Radaydeh, "Receive maximal-ratio combining with outdated arbitrary transmit antenna selection in Nakagami-m fading," IET Commun., vol. 3, no. 10, pp. 1638-1648, Oct. 2009.

[32] M. B. Neelesh, S. Kashyap, and A. F. Molisch. "Antenna selection in LTE: from motivation to specification." IEEE Commun. Mag., vol. 50, no.1, pp. 144-150, 2012. 


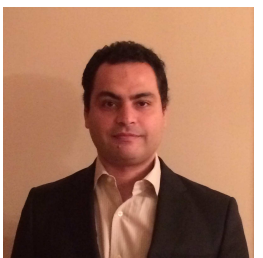

Amr A.AbdelNabi received the B.Sc. degree in Electrical Engineering from Cairo University, and M.Sc. degrees in Electrical Engineering from Nile University, Cairo, Egypt, in 2004 and 2012, respectively. From 2006 to 2007, he served as Radio planning Engineer for WCDMA networks in Vodafone Egypt. From 2007 to 2013, he served as Radio optimization Expert for WCDMA networks in Orange subsidiary (Mobinil), Egypt. In October 2013, he joined the Department of Electrical and Computer Engineering at Texas A\&M University at Qatar as Research Associate. His research interests include stochastic geometry and its application to wireless networks, channel modeling, interference modelling, MIMO communication systems, cooperative communications, device-to-device communication, applied signal processing, compressed sensing. In 2016, he joined IMDEA networks as PhD student.

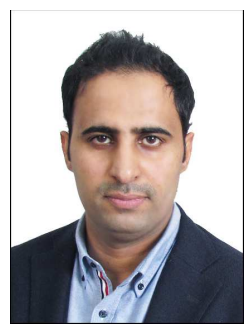

Fawaz S. Al-Qahtani received the B.Sc. degree in electrical engineering from King $\mathrm{Fa}-$ had University of Petroleum and Minerals, Dhahran, Saudi Arabia, in 2000, the M.Sc. degree in digital communication systems from Monash University, Melbourne, Australia, in 2005, and the Ph.D. degree in electrical and computer engineering from RMIT University, Melbourne, Australia, in December 2009. He is currently an Assistant Research Scientist with the Department of Electrical and Computer Engineering, Texas A\&M University at Qatar, Doha, Qatar. His current research interests include channel modeling, applied signal processing, MIMO communication systems, cooperative communications, cognitive radio systems, free-space optical, physical-layer security, visible light communication, deviceto-device communication, and power transfer. Dr. Al-Qahtani was the recipient of sponsorship from the Qatar National Research Fund as well as from JSERP and NPRP projects. He is the author and coauthor of over 70 papers in refereed mainstream journals and reputed international conferences. He was the recipient of the research excellence award from Texas A\&M University at Qatar in 2013.

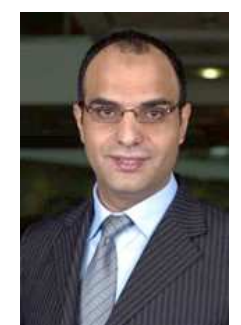

Redha M. Radaydeh (S'05, M'07, SM'13) was born in Irbid, Jordan, on November 12, 1978. He received the B.S. and M.S. degrees from Jordan University of Science and Technology (JUST), Irbid, in 2001 and 2003, respectively, and the Ph.D. degree from The University of Mississippi, Oxford, MS, USA, in 2006, all in Electrical Engineering. Previously, he worked at JUST, King Abdullah University of Science and Technology (KAUST), Texas A\&M University at Qatar (TAMUQ), and Alfaisal University, Riyadh, Saudi Arabia, as an Associate Professor of Electrical Engineering. He is now a Research Consultant with KAUST. His research interests include broad topics on wireless communications, and design and performance analysis of wireless networks.

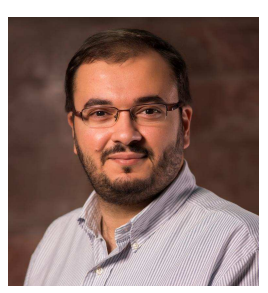

Mohammad Shaqfeh (S'07-M'09) received the B.Sc. degree in electrical engineering (communications stream) from the United Arab Emirates University in 2003 and the M.Sc. degree in communications technology from Ulm University in Germany in 2005. He received the Ph.D. degree from The University of Edinburgh, Edinburgh, Scotland, U.K., in 2009. In January 2009, he joined the Department of Electrical and Computer Engineering at Texas A\&M University at Qatar, where he is currently working as an Associate Research Scientist. His research interests include wireless communications systems, information theory and smart transportation networks. 